\title{
A New Hybrid Extragradient Iterative Method for Approximating the Common Solutions of a System of Variational Inequalities, a Mixed Equilibrium Problem, and a Hierarchical Fixed Point Problem
}

\author{
Abdellah Bnouhachem ${ }^{1,2}$ and Abdelouahed Hamdi ${ }^{3}$ \\ ${ }^{1}$ School of Management Science and Engineering, Nanjing University, Nanjing 210093, China \\ ${ }^{2}$ Ibn Zohr University, ENSA, BP 1136, Agadir, Morocco \\ ${ }^{3}$ Department of Mathematics, Statistics and Physics College of Arts and Sciences, Qatar University, P.O. Box 2713, Doha, Qatar
}

Correspondence should be addressed to Abdellah Bnouhachem; babedallah@yahoo.com and Abdelouahed Hamdi; abhamdi@qu.edu.qa

Received 31 August 2013; Accepted 4 October 2013

Academic Editor: Erdal Karapınar

Copyright (c) 2013 A. Bnouhachem and A. Hamdi. This is an open access article distributed under the Creative Commons Attribution License, which permits unrestricted use, distribution, and reproduction in any medium, provided the original work is properly cited.

We suggest and analyze an iterative scheme for finding the approximate element of the common set of solutions of a system of variational inequalities, a mixed equilibrium problem, and a hierarchical fixed point problem in a real Hilbert space. Strong convergence of the proposed method is proved under some conditions. The results presented in this paper extend and improve some well-known results in the literature.

\section{Introduction}

Let $H$ be a real Hilbert space, whose inner product and norm are denoted by $\langle\cdot, \cdot\rangle$ and $\|\cdot\|$. Let $C$ be a nonempty closed convex subset of $H$. We consider the system of variational inequalities of finding $\left(x^{*}, y^{*}\right) \in C \times C$ such that

$$
\begin{array}{ll}
\left\langle\mu_{1} B_{1} y^{*}+x^{*}-y^{*}, x-x^{*}\right\rangle \geq 0 ; & \forall x \in C, \mu_{1}>0, \\
\left\langle\mu_{2} B_{2} x^{*}+y^{*}-x^{*}, x-y^{*}\right\rangle \geq 0 ; & \forall x \in C, \mu_{2}>0,
\end{array}
$$

where $B_{i}: C \rightarrow C$ is a nonlinear mapping for each $i=1,2$. The solution set of (1) is denoted by $S^{*}$.

If $B_{1}=B_{2}=B$, then the problem (1) reduces finding $\left(x^{*}, y^{*}\right) \in C \times C$ such that

$$
\begin{array}{ll}
\left\langle\mu_{1} B y^{*}+x^{*}-y^{*}, x-x^{*}\right\rangle \geq 0 ; & \forall x \in C, \mu_{1}>0, \\
\left\langle\mu_{2} B x^{*}+y^{*}-x^{*}, x-y^{*}\right\rangle \geq 0 ; & \forall x \in C, \mu_{2}>0,
\end{array}
$$

which has been introduced and studied by Verma $[1,2]$.
If $x^{*}=y^{*}$ and $\mu_{1}=\mu_{2}$, then the problem (2) collapses to the classical variational inequality finding $x^{*} \in C$, such that

$$
\left\langle B x^{*}, x-x^{*}\right\rangle \geq 0, \quad \forall x \in C
$$

is called the classical variational inequality problem, which was introduced by Stampacchia [3] in 1964. For the recent applications, numerical techniques, and physical formulation, see [1-33]. We now have a variety of techniques to suggest and analyze various iterative algorithms for solving the system of variational inequalities (1); see $[1,2,7,8,12,14$, $24,28,30]$.

We introduce the following definitions which are useful in the following analysis.

Definition 1. The mapping $T: C \rightarrow H$ is said to be

(a) monotone, if

$$
\langle T x-T y, x-y\rangle \geq 0, \quad \forall x, y \in C ;
$$


(b) strongly monotone, if there exists an $\alpha>0$ such that

$$
\langle T x-T y, x-y\rangle \geq \alpha\|x-y\|^{2}, \quad \forall x, y \in C ;
$$

(c) $\alpha$-inverse strongly monotone, if there exists an $\alpha>0$ such that

$$
\langle T x-T y, x-y\rangle \geq \alpha\|T x-T y\|^{2}, \quad \forall x, y \in C ;
$$

(d) nonexpansive, if

$$
\|T x-T y\| \leq\|x-y\|, \quad \forall x, y \in C ;
$$

(e) $k$-Lipschitz continuous, if there exists a constant $k>0$ such that

$$
\|T x-T y\| \leq k\|x-y\|, \quad \forall x, y \in C ;
$$

(f) contraction on $C$, if there exists a constant $0 \leq k<1$ such that

$$
\|T x-T y\| \leq k\|x-y\|, \quad \forall x, y \in C .
$$

It is easy to observe that every $\alpha$-inverse strongly monotone $T$ is monotone and Lipschitz continuous. A mapping $T$ : $C \rightarrow H$ is called $k$-strict pseudocontraction, if there exists a constant $0 \leq k<1$ such that

$$
\begin{array}{r}
\|T x-T y\|^{2} \leq\|x-y\|^{2}+k\|(I-T) x-(I-T) y\|^{2}, \\
\forall x, y \in C .
\end{array}
$$

The fixed-point problem for the mapping $T$ is to find $x \in C$ such that

$$
T x=x .
$$

We denote by $F(T)$ the set of solutions of (11). It is well-known that the class of strict pseudocontractions strictly includes the class of nonexpansive mappings; then $F(T)$ is closed and convex and $P_{F(T)}$ is well defined (see [33]).

The mixed equilibrium problem, denoted by MEP, is to find $x \in C$ such that

$$
F(x, y)+\langle D x, y-x\rangle \geq 0, \quad \forall y \in C,
$$

where $F: C \times C \rightarrow \mathbb{R}$ is bifunction and $D: C \rightarrow H$ is a nonlinear mapping. This problem was introduced and studied by Moudafi and Théra [21] and Moudafi [22]. The set of solutions of (12) is denoted by

$$
\operatorname{MEP}(F):=\{x \in C: F(x, y)+\langle D x, y-x\rangle \geq 0, \forall y \in C\} .
$$

If $D=0$, then it is reduced to the equilibrium problem is to find $x \in C$ such that

$$
F(x, y) \geq 0, \quad \forall y \in C .
$$

The solution set of (14) is denoted by $\operatorname{EP}(F)$. Numerous problems in physics, optimization, and economics reduce to find a solution of (14); see $[9,13,25,26]$. In 1997, Flåm and Antipin [10] introduced an iterative scheme of finding the best approximation to the initial data when $\mathrm{EP}(F)$ is nonempty. Recently, Plubtieng and Punpaeng [25] introduced an iterative method for finding the common element of the set $F(T) \cap \Omega^{*} \cap \operatorname{EP}(F)$.

Let $S: C \rightarrow H$ be a nonexpansive mapping. The following problem is called a hierarchical fixed point problem: Find $x \in F(T)$ such that

$$
\langle x-S x, y-x\rangle \geq 0, \quad \forall y \in F(T) .
$$

It is known that the hierarchical fixed-point problem (15) links with some monotone variational inequalities and convex programming problems; see [11, 31]. Various methods have been proposed to solve the hierarchical fixed point problem; see Moudafi [23], Maingé and Moudafi in [17], Marino and $\mathrm{Xu}$ in [19], and Cianciaruso et al. [6]. Very recently, Yao et al. [31] introduced the following strong convergence iterative algorithm to solve the problem (15):

$$
\begin{gathered}
y_{n}=\beta_{n} S x_{n}+\left(1-\beta_{n}\right) x_{n} \\
x_{n+1}=P_{C}\left[\alpha_{n} f\left(x_{n}\right)+\left(1-\alpha_{n}\right) T y_{n}\right], \quad \forall n \geq 0,
\end{gathered}
$$

where $f: C \rightarrow H$ is a contraction mapping and $\left\{\alpha_{n}\right\}$ and $\left\{\beta_{n}\right\}$ are two sequences in $(0,1)$. Under some certain restrictions on parameters, Yao et al. proved that the sequence $\left\{x_{n}\right\}$ generated by (16) converges strongly to $z \in F(T)$, which is the unique solution of the following variational inequality:

$$
\langle(I-f) z, y-z\rangle \geq 0, \quad \forall y \in F(T) .
$$

By changing the restrictions on parameters, the authors obtained another result on the iterative scheme (16); the sequence $\left\{x_{n}\right\}$ generated by (16) converges strongly to a point $z \in F(T)$, which is the unique solution of the following variational inequality:

$$
\left\langle\frac{1}{\tau}(I-f) z+(I-S) z, y-z\right\rangle \geq 0, \quad \forall y \in F(T) .
$$

Let $S: C \rightarrow H$ be a nonexpansive mapping and $\left\{T_{i}\right\}_{i=1}^{\infty}$ : $C \rightarrow C$ a countable family of nonexpansive mappings. Very recently, Gu et al. [11] introduced the following iterative algorithm:

$$
\begin{gathered}
y_{n}=P_{C}\left[\beta_{n} S x_{n}+\left(1-\beta_{n}\right) x_{n}\right] \\
x_{n+1}=P_{C}\left[\alpha_{n} f\left(x_{n}\right)+\sum_{i=1}^{n}\left(\alpha_{i-1}-\alpha_{i}\right) T_{i} y_{n}\right], \quad \forall n \geq 1,
\end{gathered}
$$

where $\alpha_{0}=1,\left\{\alpha_{n}\right\}$ is a strictly decreasing sequence in $(0,1)$, and $\left\{\beta_{n}\right\}$ is a sequence in $(0,1)$. Under some certain conditions on parameters, $\mathrm{Gu}$ et al. proved that the sequence $\left\{x_{n}\right\}$ generated by (19) converges strongly to $z \in \bigcap_{i=1}^{\infty} F\left(T_{i}\right)$, which is unique solution of one of the variational inequalities (17) and (18).

In this paper, motivated by the work of Yao et al. [31] and $\mathrm{Gu}$ et al. [11] and by the recent work going in this direction, we 
give an iterative method for finding the approximate element of the common set of solutions of (1), (12), and (15) for a strictly pseudocontraction mapping in real Hilbert space. We establish a strong convergence theorem based on this method. The presented method improves and generalizes many known results for solving system of variational inequality problems, mixed equilibrium problems, and hierarchical fixed point problems; see, for example $[6,11,17,31]$ and relevant references cited therein.

\section{Preliminaries}

In this section, we list some fundamental lemmas that are useful in the consequent analysis. The first lemma provides some basic properties of projection onto $C$.

Lemma 2. Let $P_{C}$ denote the projection of $H$ onto $C$. Then, one has the following inequalities:

$$
\begin{array}{r}
\left\langle z-P_{C}[z], P_{C}[z]-v\right\rangle \geq 0, \quad \forall z \in H, v \in C ; \\
\left\langle u-v, P_{C}[u]-P_{C}[v]\right\rangle \geq\left\|P_{C}[u]-P_{C}[v]\right\|^{2}, \\
\forall u, v \in H ; \\
\left\|P_{C}[u]-P_{C}[v]\right\| \leq u-v \|, \quad \forall u, v \in H ; \\
\left\|u-P_{C}[z]\right\|^{2} \leq\|z-u\|^{2}-\left\|z-P_{C}[z]\right\|^{2}, \\
\forall z \in H, u \in C .
\end{array}
$$

Lemma 3 (see [7]). For any $\left(x^{*}, y^{*}\right) \in C \times C,\left(x^{*}, y^{*}\right)$ is a solution of (1) if and only if $x^{*}$ is a fixed point of the mapping $Q: C \rightarrow C$ de fined by

$$
\begin{array}{r}
Q(x)=P_{C}\left[P_{C}\left[x-\mu_{2} B_{2} x\right]-\mu_{1} B_{1} P_{C}\left[x-\mu_{2} B_{2} x\right]\right], \\
\forall x \in C,
\end{array}
$$

where $y^{*}=P_{C}\left[x^{*}-\mu_{2} B_{2} x^{*}\right], \mu_{i} \in\left(0,2 \theta_{i}\right)$, and $B_{i}: C \rightarrow C$ is $\theta_{i}$-inverse strongly monotone mappings for each $i=1,2$.

Lemma 4 (see [5]). Let $F: C \times C \rightarrow \mathbb{R}$ be a bifunction satisfying the following assumptions.

(i) $F(x, x)=0, \forall x \in C$.

(ii) $F$ is monotone; that is, $F(x, y)+F(y, x) \leq 0, \forall x, y \in C$.

(iii) For each $x, y, z \in C, \lim _{t \rightarrow 0} F(t z+(1-t) x, y) \leq$ $F(x, y)$.

(iv) For each $x \in C, y \rightarrow F(x, y)$ is convex and lower semicontinuous.

Let $r>0$ and $x \in H$. Then, there exists $z \in C$ such that

$$
F(z, y)+\frac{1}{r}\langle y-z, z-x\rangle \geq 0, \quad \forall y \in C .
$$

Lemma 5 (see [10]). Assume that $F: C \times C \rightarrow \mathbb{R}$ satisfies assumptions (i)-(iv) of Lemma 4. For $r>0$ and forall $x \in H$, define a mapping $T_{r}: H \rightarrow C$ as follows:

$$
T_{r}(x)=\left\{z \in C: F(z, y)+\frac{1}{r}\langle y-z, z-x\rangle \geq 0, \forall y \in C\right\} .
$$

Then, the following hold.

(i) $T_{r}$ is single valued.

(ii) $T_{r}$ is firmly nonexpansive; that is,

$\left\|T_{r} x-T_{r} y\right\|^{2} \leq\left\langle T_{r} x-T_{r} y, x-y\right\rangle, \quad \forall x, y \in H$.

(iii) $F\left(T_{r}\right)=\operatorname{EP}(F)$.

(iv) $\mathrm{EP}(F)$ is closed and convex.

Lemma 6 (see [32]). Let $C$ be a nonempty closed convex subset of a real Hilbert space $H$. If $T: C \rightarrow C$ is a $k$-strict pseudocontraction, then

(i) the mapping $I-T$ is demiclosed at 0 ; that is, if $\left\{x_{n}\right\}$ is a sequence in $C$ weakly converging to $x$ and if $\left\{(I-T) x_{n}\right\}$ converges strongly to 0 , then $(I-T) x=0$;

(ii) the set $F(T)$ of $T$ is closed and convex so that the projection $P_{F(T)}$ is well defined.

Lemma 7 (see [29]). Assume that $\left\{a_{n}\right\}$ is a sequence of nonnegative real numbers such that

$$
a_{n+1} \leq\left(1-\gamma_{n}\right) a_{n}+\delta_{n}
$$

where $\left\{\gamma_{n}\right\}$ is a sequence in $(0,1)$ and $\delta_{n}$ is a sequence such that

(1) $\sum_{n=1}^{\infty} \gamma_{n}=\infty$;

(2) $\lim \sup _{n \rightarrow \infty} \delta_{n} / \gamma_{n} \leq 0$ or $\sum_{n=1}^{\infty}\left|\delta_{n}\right|<\infty$.

Then, $\lim _{n \rightarrow \infty} a_{n}=0$.

Lemma 8 (see [4]). Let $C$ be a closed convex subset of $H$. Let $\left\{x_{n}\right\}$ be a bounded sequence in $H$. Assume that

(i) the weak $w$-limit set $w_{w}\left(x_{n}\right) \subset C$ where $w_{w}\left(x_{n}\right)=\{x$ : $\left.x_{n_{i}} \rightarrow x\right\}$,

(ii) for each $z \in C, \lim _{n \rightarrow \infty}\left\|x_{n}-z\right\|$ exists.

Then, $\left\{x_{n}\right\}$ is weakly convergent to a point in $C$.

Lemma 9 (see [33]). Let $H$ be a Hilbert space, $C$ a closed and convex subset of $H$, and $T: C \rightarrow C$ a $k$-strict pseudocontraction mapping. Define a mapping $V: C \rightarrow H$ by $V x=\lambda x+(1-\lambda) T x$, for all $x \in C$. Then, as $k \leq \lambda<1, V$ is a nonexpansive mapping such that $F(V)=F(T)$.

Lemma 10 (see [11]). Let $H$ be a Hilbert space, $C$ a closed and convex subset of $H$, and $T: C \rightarrow C$ a nonexpansive mapping such that $F(T) \neq \emptyset$. Then,

$$
\|T x-x\|^{2} \leq 2\left\langle x-T x, x-x^{\prime}\right\rangle, \quad \forall x^{\prime} \in F(T), \forall x \in C .
$$

\section{The Proposed Method and Some Properties}

In this section, we suggest and analyze our method for finding the common solutions of the system of variational inequality problem (1), the mixed equilibrium problem (12), and the hierarchical fixed point problem (15). 
Let $C$ be a nonempty closed convex subset of a real Hilbert space $H$. Let $D, B_{i}: C \rightarrow H$ be $\eta, \theta_{i}$-inverse strongly monotone mappings for each $i=1,2$, respectively. Let $F: C \times$ $C \rightarrow \mathbb{R}$ be a bifunction satisfying the assumptions (i)-(iv) of Lemma 4, $S: C \rightarrow H$ a nonexpansive mapping, and $\left\{T_{i}\right\}_{i=1}^{\infty}$ : $C \rightarrow C$ a countable family of $k_{i}$-strict pseudocontraction mappings such that $S^{*} \cap \operatorname{MEP}(F) \cap F(T) \neq \emptyset$, where $F(T):=$ $\bigcap_{i=1}^{\infty} F\left(T_{i}\right)=\bigcap_{i=1}^{\infty} F\left(V_{i}\right)$. Let $f$ be a $\rho$-contraction mapping.

Algorithm 11. For a given $x_{0} \in C$ arbitrarily, let the iterative sequences $\left\{u_{n}\right\},\left\{x_{n}\right\},\left\{y_{n}\right\}$, and $\left\{z_{n}\right\}$ be generated by

$$
\begin{array}{r}
F\left(u_{n}, y\right)+\left\langle D x_{n}, y-u_{n}\right\rangle+\frac{1}{r_{n}}\left\langle y-u_{n}, u_{n}-x_{n}\right\rangle \geq 0, \\
\forall y \in C ; \\
z_{n}=P_{C}\left[P_{C}\left[u_{n}-\mu_{2} B_{2} u_{n}\right]-\mu_{1} B_{1} P_{C}\left[u_{n}-\mu_{2} B_{2} u_{n}\right]\right] ; \\
y_{n}=P_{C}\left[\beta_{n} S x_{n}+\left(1-\beta_{n}\right) z_{n}\right] ; \\
x_{n+1}=P_{C}\left[\alpha_{n} f\left(x_{n}\right)+\sum_{i=1}^{n}\left(\alpha_{i-1}-\alpha_{i}\right) V_{i} y_{n}\right], \quad \forall n \geq 0,
\end{array}
$$

where $V_{i}=k_{i} I+\left(1-k_{i}\right) T_{i}, 0 \leq k_{i}<1, \mu_{i} \in\left(0,2 \theta_{i}\right)$ for each $i=1,2,\left\{r_{n}\right\} \subset(0,2 \eta), \alpha_{0}=1,\left\{\alpha_{n}\right\}$ is a strictly decreasing sequence in $(0,1)$, and $\left\{\beta_{n}\right\}$ is a sequence in $(0,1)$ satisfying the following conditions:

(a) $\lim _{n \rightarrow \infty} \alpha_{n}=0$ and $\sum_{n=1}^{\infty} \alpha_{n}=\infty$,

(b) $\lim _{n \rightarrow \infty}\left(\beta_{n} / \alpha_{n}\right)=0$,

(c) $\sum_{n=1}^{\infty}\left|\alpha_{n-1}-\alpha_{n}\right|<\infty$ and $\sum_{n=1}^{\infty}\left|\beta_{n-1}-\beta_{n}\right|<\infty$,

(d) $\lim _{\inf _{n \rightarrow \infty}} r_{n}<\limsup _{n \rightarrow \infty} r_{n}<2 \eta$ and $\sum_{n=1}^{\infty}\left|r_{n-1}-r_{n}\right|<\infty$.

Lemma 12. Let $x^{*} \in S^{*} \cap \operatorname{MEP}(F) \cap F(T)$. Then, $\left\{x_{n}\right\},\left\{u_{n}\right\}$, $\left\{z_{n}\right\}$, and $\left\{y_{n}\right\}$ are bounded.

Proof. First, we show that the mapping $\left(I-r_{n} D\right)$ is nonexpansive. For any $x, y \in C$,

$$
\begin{aligned}
\left\|\left(I-r_{n} D\right) x-\left(I-r_{n} D\right) y\right\|^{2}= & \left\|(x-y)-r_{n}(D x-D y)\right\|^{2} \\
= & \|x-y\|^{2} \\
& -2 r_{n}\langle x-y, D x-D y\rangle \\
& +r_{n}^{2}\|D x-D y\|^{2} \leq\|x-y\|^{2} \\
& -r_{n}\left(2 \eta-r_{n}\right)\|D x-D y\|^{2} \\
\leq & \|x-y\|^{2} .
\end{aligned}
$$

Similarly, we can show that the mapping $\left(I-\mu_{i} B_{i}\right)$ is nonexpansive for each $i=1,2$. It follows from Lemma 5 that
$u_{n}=T_{r_{n}}\left(x_{n}-r_{n} D x_{n}\right)$. Let $x^{*} \in S^{*} \cap \operatorname{MEP}(F) \cap F(T)$; we have $x^{*}=T_{r_{n}}\left(x^{*}-r_{n} D x^{*}\right)$, and it follows that

$$
\begin{aligned}
\left\|u_{n}-x^{*}\right\|^{2} & =\left\|T_{r_{n}}\left(x_{n}-r_{n} D x_{n}\right)-T_{r_{n}}\left(x^{*}-r_{n} D x^{*}\right)\right\|^{2} \\
& \leq\left\|\left(x_{n}-r_{n} D x_{n}\right)-\left(x^{*}-r_{n} D x^{*}\right)\right\|^{2} \\
& \leq\left\|x_{n}-x^{*}\right\|^{2}-r_{n}\left(2 \eta-r_{n}\right)\left\|D x_{n}-D x^{*}\right\|^{2} \\
& \leq\left\|x_{n}-x^{*}\right\|^{2} .
\end{aligned}
$$

Let $x^{*} \in S^{*} \cap \operatorname{MEP}(F) \cap F(T)$; we have

$$
x^{*}=P_{C}\left[y^{*}-\mu_{1} B_{1} y^{*}\right],
$$

where

$$
y^{*}=P_{C}\left[x^{*}-\mu_{2} B_{2} x^{*}\right] .
$$

Setting $v_{n}=P_{C}\left[u_{n}-\mu_{2} B_{2} u_{n}\right]$. Since $B_{2}$ is $\theta_{2}$-inverse strongly monotone mapping, it follows that

$$
\begin{aligned}
\left\|v_{n}-y^{*}\right\|^{2} & =\left\|P_{C}\left[u_{n}-\mu_{2} B_{2} u_{n}\right]-P_{C}\left[x^{*}-\mu_{2} B_{2} x^{*}\right]\right\|^{2} \\
& \leq\left\|u_{n}-u^{*}-\mu_{2}\left(B_{2} u_{n}-B_{2} x^{*}\right)\right\|^{2} \\
& \leq\left\|x_{n}-x^{*}\right\|^{2}-\mu_{2}\left(2 \theta-\mu_{2}\right)\left\|B_{2} u_{n}-B_{2} x^{*}\right\|^{2} \\
& \leq\left\|x_{n}-x^{*}\right\|^{2} .
\end{aligned}
$$

Since $B_{i}$ is $\theta_{i}$-inverse strongly monotone mappings for each $i=1,2$, we get

$$
\begin{gathered}
\left\|z_{n}-x^{*}\right\|^{2} \\
=\| P_{C}\left[P_{C}\left[u_{n}-\mu_{2} B_{2} u_{n}\right]-\mu_{1} B_{1} P_{C}\left[u_{n}-\mu_{2} B_{2} u_{n}\right]\right] \\
\quad-P_{C}\left[P_{C}\left[x^{*}-\mu_{2} B_{2} x^{*}\right]\right. \\
\left.\quad-\mu_{1} B_{1} P_{C}\left[x^{*}-\mu_{2} B_{2} x^{*}\right]\right] \|^{2} \\
\leq \| P_{C}\left[u_{n}-\mu_{2} B_{2} u_{n}\right]-\mu_{1} B_{1} P_{C}\left[u_{n}-\mu_{2} B_{2} u_{n}\right] \\
\quad-\left(P_{C}\left[x^{*}-\mu_{2} B_{2} x^{*}\right]-\mu_{1} B_{1} P_{C}\left[x^{*}-\mu_{2} B_{2} x^{*}\right]\right) \|^{2} \\
=\| P_{C}\left[u_{n}-\mu_{2} B_{2} u_{n}\right]-P_{C}\left[x^{*}-\mu_{2} B_{2} x^{*}\right] \\
\quad-\mu_{1}\left(B_{1} P_{C}\left[u_{n}-\mu_{2} B_{2} u_{n}\right]-B_{1} P_{C}\left[x^{*}-\mu_{2} B_{2} x^{*}\right]\right) \|^{2} \\
\leq\left\|P_{C}\left[u_{n}-\mu_{2} B_{2} u_{n}\right]-P_{C}\left[x^{*}-\mu_{2} B_{2} x^{*}\right]\right\|^{2} \\
\quad-\mu_{1}\left(2 \theta_{1}-\mu_{1}\right) \| B_{1} P_{C}\left[u_{n}-\mu_{2} B_{2} u_{n}\right] \\
\quad-B_{1} P_{C}\left[x^{*}-\mu_{2} B_{2} x^{*}\right] \|^{2}
\end{gathered}
$$




$$
\begin{aligned}
& \leq\left\|\left(u_{n}-\mu_{2} B_{2} u_{n}\right)-\left(x^{*}-\mu_{2} B_{2} x^{*}\right)\right\|^{2} \\
& -\mu_{1}\left(2 \theta_{1}-\mu_{1}\right) \| B_{1} P_{C}\left[u_{n}-\mu_{2} B_{2} u_{n}\right] \\
& \quad-B_{1} P_{C}\left[x^{*}-\mu_{2} B_{2} x^{*}\right] \|^{2} \\
& \leq\left\|u_{n}-x^{*}\right\|^{2}-\mu_{2}\left(2 \theta_{2}-\mu_{2}\right)\left\|B_{2} u_{n}-B_{2} x^{*}\right\|^{2} \\
& \quad-\mu_{1}\left(2 \theta_{1}-\mu_{1}\right)\left\|B_{1} v_{n}-B_{1} y^{*}\right\|^{2} \\
& \leq\left\|u_{n}-x^{*}\right\|^{2} \leq\left\|x_{n}-x^{*}\right\|^{2} .
\end{aligned}
$$

Next, we prove that the sequence $\left\{x_{n}\right\}$ is bounded; without loss of generality we can assume that $\beta_{n} \leq \alpha_{n}$ for all $n \geq 1$. From (29), we have

$$
\begin{aligned}
& \left\|x_{n+1}-x^{*}\right\| \leq \| \alpha_{n} f\left(x_{n}\right)+\sum_{i=1}^{n}\left(\alpha_{i-1}-\alpha_{i}\right) V_{i} y_{n} \\
& -\alpha_{n} x^{*}-\sum_{i=1}^{n}\left(\alpha_{i-1}-\alpha_{i}\right) V_{i} x^{*} \| \\
& \leq \alpha_{n}\left\|f\left(x_{n}\right)-f\left(x^{*}\right)\right\|+\alpha_{n}\left\|f\left(x^{*}\right)-x^{*}\right\| \\
& +\sum_{i=1}^{n}\left(\alpha_{i-1}-\alpha_{i}\right)\left\|V_{i} y_{n}-V_{i} x^{*}\right\| \\
& \leq \alpha_{n}\left\|f\left(x_{n}\right)-f\left(x^{*}\right)\right\|+\alpha_{n}\left\|f\left(x^{*}\right)-x^{*}\right\| \\
& +\sum_{i=1}^{n}\left(\alpha_{i-1}-\alpha_{i}\right)\left\|y_{n}-x^{*}\right\| \\
& \leq \alpha_{n}\left\|f\left(x_{n}\right)-f\left(x^{*}\right)\right\|+\alpha_{n}\left\|f\left(x^{*}\right)-x^{*}\right\| \\
& +\left(1-\alpha_{n}\right)\left\|\beta_{n} S x_{n}+\left(1-\beta_{n}\right) z_{n}-x^{*}\right\| \\
& \leq \alpha_{n}\left\|f\left(x_{n}\right)-f\left(x^{*}\right)\right\|+\alpha_{n}\left\|f\left(x^{*}\right)-x^{*}\right\| \\
& +\left(1-\alpha_{n}\right)\left(\beta_{n}\left\|S x_{n}-S x^{*}\right\|+\beta_{n}\left\|S x^{*}-x^{*}\right\|\right. \\
& \left.+\left(1-\beta_{n}\right)\left\|z_{n}-x^{*}\right\|\right) \\
& \leq \alpha_{n} \rho\left\|x_{n}-x^{*}\right\|+\alpha_{n}\left\|f\left(x^{*}\right)-x^{*}\right\| \\
& +\left(1-\alpha_{n}\right)\left(\beta_{n}\left\|x_{n}-x^{*}\right\|+\beta_{n}\left\|S x^{*}-x^{*}\right\|\right. \\
& \left.+\left(1-\beta_{n}\right)\left\|x_{n}-x^{*}\right\|\right) \\
& =\left(1-\alpha_{n}(1-\rho)\right) \\
& \times\left\|x_{n}-x^{*}\right\|+\alpha_{n}\left\|f\left(x^{*}\right)-x^{*}\right\| \\
& +\left(1-\alpha_{n}\right) \beta_{n}\left\|S x^{*}-x^{*}\right\| \leq\left(1-\alpha_{n}(1-\rho)\right) \\
& \times\left\|x_{n}-x^{*}\right\|+\alpha_{n}\left\|f\left(x^{*}\right)-x^{*}\right\| \\
& +\beta_{n}\left\|S x^{*}-x^{*}\right\| \\
& \leq\left(1-\alpha_{n}(1-\rho)\right)\left\|x_{n}-x^{*}\right\| \\
& +\alpha_{n}\left(\left\|f\left(x^{*}\right)-x^{*}\right\|+\left\|S x^{*}-x^{*}\right\|\right)
\end{aligned}
$$

$$
\begin{aligned}
& =\left(1-\alpha_{n}(1-\rho)\right)\left\|x_{n}-x^{*}\right\|+\frac{\alpha_{n}(1-\rho)}{1-\rho} \\
& \times\left(\left\|f\left(x^{*}\right)-x^{*}\right\|+\left\|S x^{*}-x^{*}\right\|\right) \\
& \leq \max \left\{\left\|x_{n}-x^{*}\right\|, \frac{1}{1-\rho}\left(\left\|f\left(x^{*}\right)-x^{*}\right\|\right.\right. \\
& \left.\left.+\left\|S x^{*}-x^{*}\right\|\right)\right\} .
\end{aligned}
$$

By induction on $n$, we obtain $\left\|x_{n}-x^{*}\right\| \leq \max \left\{\| x_{0}-\right.$ $\left.x^{*} \|,(1 /(1-\rho))\left(\left\|f\left(x^{*}\right)-x^{*}\right\|+\left\|S x^{*}-x^{*}\right\|\right)\right\}$, for $n \geq 0$ and $x_{0} \in C$. Hence, $\left\{x_{n}\right\}$ is bounded and, consequently, we deduce that $\left\{u_{n}\right\},\left\{z_{n}\right\},\left\{v_{n}\right\}$, and $\left\{y_{n}\right\}$ are bounded.

Lemma 13. Let $x^{*} \in S^{*} \cap \operatorname{MEP}(F) \cap F(T)$ and $\left\{x_{n}\right\}$ be the sequence generated by Algorithm 11. Then one has

(a) $\lim _{n \rightarrow \infty}\left\|x_{n+1}-x_{n}\right\|=0$.

(b) The weak $w$-limit set $w_{w}\left(x_{n}\right) \subset F(T),\left(w_{w}\left(x_{n}\right)=\{x\right.$ : $\left.\left.x_{n_{i}}-x\right\}\right)$.

Proof. Since $u_{n}=T_{r_{n}}\left(x_{n}-r_{n} D x_{n}\right)$ and $u_{n-1}=T_{r_{n-1}}\left(x_{n-1}-\right.$ $\left.r_{n-1} D x_{n-1}\right)$, we have

$$
\begin{array}{cr}
F\left(u_{n}, y\right)+\left\langle D x_{n}, y-u_{n}\right\rangle+\frac{1}{r_{n}}\left\langle y-u_{n}, u_{n}-x_{n}\right\rangle \geq 0, & \forall y \in C, \\
F\left(u_{n-1}, y\right)+\left\langle D x_{n-1}, y-u_{n-1}\right\rangle & \\
+\frac{1}{r_{n-1}}\left\langle y-u_{n-1}, u_{n-1}-x_{n-1}\right\rangle \geq 0, &
\end{array}
$$

$\forall y \in C$.

Take $y=u_{n-1}$ in (37) and $y=u_{n}$ in (38), we get

$$
\begin{gathered}
F\left(u_{n}, u_{n-1}\right)+\left\langle D x_{n}, u_{n-1}-u_{n}\right\rangle \\
+\frac{1}{r_{n}}\left\langle u_{n-1}-u_{n}, u_{n}-x_{n}\right\rangle \geq 0, \\
F\left(u_{n-1}, u_{n}\right)+\left\langle D x_{n-1}, u_{n}-u_{n-1}\right\rangle \\
+\frac{1}{r_{n-1}}\left\langle u_{n}-u_{n-1}, u_{n-1}-x_{n-1}\right\rangle \geq 0 .
\end{gathered}
$$

Adding (39) and using the monotonicity of $F$, we have

$$
\begin{aligned}
\left\langle D x_{n-1}\right. & \left.-D x_{n}, u_{n}-u_{n-1}\right\rangle \\
& +\left\langle u_{n}-u_{n-1}, \frac{u_{n-1}-x_{n-1}}{r_{n-1}}-\frac{u_{n}-x_{n}}{r_{n}}\right\rangle \geq 0,
\end{aligned}
$$


which implies that

$$
\begin{aligned}
& 0 \leq\left\langle u_{n}-u_{n-1}, r_{n}\left(D x_{n-1}-D x_{n}\right)+\frac{r_{n}}{r_{n-1}}\left(u_{n-1}-x_{n-1}\right)\right. \\
& \left.-\left(u_{n}-x_{n}\right)\right\rangle \\
& =\left\langle u_{n-1}-u_{n}, u_{n}-u_{n-1}\right. \\
& +\left(1-\frac{r_{n}}{r_{n-1}}\right) u_{n-1}+\left(x_{n-1}-r_{n} D x_{n-1}\right) \\
& \left.-\left(x_{n}-r_{n} D x_{n}\right)-x_{n-1}+\frac{r_{n}}{r_{n-1}} x_{n-1}\right\rangle \\
& =\left\langle u_{n-1}-u_{n},\left(1-\frac{r_{n}}{r_{n-1}}\right) u_{n-1}+\left(x_{n-1}-r_{n} D x_{n-1}\right)\right. \\
& \left.-\left(x_{n}-r_{n} D x_{n}\right)-x_{n-1}+\frac{r_{n}}{r_{n-1}} x_{n-1}\right\rangle-\left\|u_{n}-u_{n-1}\right\|^{2} \\
& =\left\langle u_{n-1}-u_{n},\left(1-\frac{r_{n}}{r_{n-1}}\right)\left(u_{n-1}-x_{n-1}\right)\right. \\
& \left.+\left(x_{n-1}-r_{n} D x_{n-1}\right)-\left(x_{n}-r_{n} D x_{n}\right)\right\rangle \\
& -\left\|u_{n}-u_{n-1}\right\|^{2} \\
& \leq\left\|u_{n-1}-u_{n}\right\| \\
& \times\left\{\left|1-\frac{r_{n}}{r_{n-1}}\right|\left\|u_{n-1}-x_{n-1}\right\|\right. \\
& \left.+\left\|\left(x_{n-1}-r_{n} D x_{n-1}\right)-\left(x_{n}-r_{n} D x_{n}\right)\right\|\right\} \\
& -\left\|u_{n}-u_{n-1}\right\|^{2} \\
& \leq\left\|u_{n-1}-u_{n}\right\| \\
& \times\left\{\left|1-\frac{r_{n}}{r_{n-1}}\right|\left\|u_{n-1}-x_{n-1}\right\|+\left\|x_{n-1}-x_{n}\right\|\right\} \\
& -\left\|u_{n}-u_{n-1}\right\|^{2}
\end{aligned}
$$

and then

$$
\begin{aligned}
\left\|u_{n-1}-u_{n}\right\| \leq & \left|1-\frac{r_{n}}{r_{n-1}}\right|\left\|u_{n-1}-x_{n-1}\right\| \\
& +\left\|x_{n-1}-x_{n}\right\| .
\end{aligned}
$$

Without loss of generality, let us assume that there exists a real number $\mu$ such that $r_{n}>\mu>0$, for all positive integers $n$. Then, we get

$$
\begin{aligned}
\left\|u_{n-1}-u_{n}\right\| \leq & \left\|x_{n-1}-x_{n}\right\| \\
& +\frac{1}{\mu}\left|r_{n-1}-r_{n}\right|\left\|u_{n-1}-x_{n-1}\right\| .
\end{aligned}
$$

Next, we estimate

$$
\begin{aligned}
& \left\|z_{n}-z_{n-1}\right\|^{2} \\
& =\| P_{C}\left[P_{C}\left[u_{n}-\mu_{2} B_{2} u_{n}\right]-\mu_{1} B_{1} P_{C}\left[u_{n}-\mu_{2} B_{2} u_{n}\right]\right] \\
& -P_{C}\left[P_{C}\left[u_{n-1}-\mu_{2} B_{2} u_{n-1}\right]\right. \\
& \left.-\mu_{1} B_{1} P_{C}\left[u_{n-1}-\mu_{2} B_{2} u_{n-1}\right]\right] \|^{2} \\
& \leq \| P_{C}\left[u_{n}-\mu_{2} B_{2} u_{n}\right]-\mu_{1} B_{1} P_{C}\left[u_{n}-\mu_{2} B_{2} u_{n}\right] \\
& -\left(P_{C}\left[u_{n-1}-\mu_{2} B_{2} u_{n-1}\right]\right. \\
& \left.-\mu_{1} B_{1} P_{C}\left[u_{n-1}-\mu_{2} B_{2} u_{n-1}\right]\right) \|^{2} \\
& =\| P_{C}\left[u_{n}-\mu_{2} B_{2} u_{n}\right]-P_{C}\left[u_{n-1}-\mu_{2} B_{2} u_{n-1}\right] \\
& -\mu_{1}\left(B_{1} P_{C}\left[u_{n}-\mu_{2} B_{2} u_{n}\right]\right. \\
& \left.-B_{1} P_{C}\left[u_{n-1}-\mu_{2} B_{2} u_{n-1}\right]\right) \|^{2} \\
& \leq\left\|P_{C}\left[u_{n}-\mu_{2} B_{2} u_{n}\right]-P_{C}\left[u_{n-1}-\mu_{2} B_{2} u_{n-1}\right]\right\|^{2} \\
& -\mu_{1}\left(2 \theta_{1}-\mu_{1}\right) \\
& \times \| B_{1} P_{C}\left[u_{n}-\mu_{2} B_{2} u_{n}\right] \\
& -B_{1} P_{C}\left[u_{n-1}-\mu_{2} B_{2} u_{n-1}\right] \|^{2} \\
& \leq\left\|P_{C}\left[u_{n}-\mu_{2} B_{2} u_{n}\right]-P_{C}\left[u_{n-1}-\mu_{2} B_{2} u_{n-1}\right]\right\|^{2} \\
& \leq\left\|\left(u_{n}-u_{n-1}\right)-\mu_{2}\left(B_{2} u_{n}-B_{2} u_{n-1}\right)\right\|^{2} \\
& \leq\left\|u_{n}-u_{n-1}\right\|^{2}-\mu_{2}\left(2 \theta_{2}-\mu_{2}\right)\left\|B_{2} u_{n}-B_{2} u_{n-1}\right\|^{2} \\
& \leq\left\|u_{n}-u_{n-1}\right\|^{2} \text {. }
\end{aligned}
$$

It follows from (43) and (44) that

$$
\begin{aligned}
\left\|z_{n}-z_{n-1}\right\| \leq & \left\|x_{n-1}-x_{n}\right\| \\
& +\frac{1}{\mu}\left|r_{n-1}-r_{n}\right|\left\|u_{n-1}-x_{n-1}\right\| .
\end{aligned}
$$

From (29) and the previous inequality, we get

$$
\begin{aligned}
\| y_{n}- & y_{n-1} \| \\
\leq & \left\|\beta_{n} S x_{n}+\left(1-\beta_{n}\right) z_{n}-\left(\beta_{n-1} S x_{n-1}+\left(1-\beta_{n-1}\right) z_{n-1}\right)\right\| \\
= & \| \beta_{n}\left(S x_{n}-S x_{n-1}\right)+\left(\beta_{n}-\beta_{n-1}\right) S x_{n-1} \\
& +\left(1-\beta_{n}\right)\left(z_{n}-z_{n-1}\right)+\left(\beta_{n-1}-\beta_{n}\right) z_{n-1} \| \\
\leq & \beta_{n}\left\|x_{n}-x_{n-1}\right\|+\left(1-\beta_{n}\right)\left\|z_{n}-z_{n-1}\right\| \\
& +\left|\beta_{n}-\beta_{n-1}\right|\left(\left\|S x_{n-1}\right\|+\left\|z_{n-1}\right\|\right) \\
\leq & \beta_{n}\left\|x_{n}-x_{n-1}\right\|+\left(1-\beta_{n}\right)
\end{aligned}
$$




$$
\begin{aligned}
& \quad \times\left\{\left\|x_{n-1}-x_{n}\right\|+\frac{1}{\mu}\left|r_{n-1}-r_{n}\right|\left\|u_{n-1}-x_{n-1}\right\|\right\} \\
& \quad+\left|\beta_{n}-\beta_{n-1}\right|\left(\left\|S x_{n-1}\right\|+\left\|z_{n-1}\right\|\right) \\
& \leq\left\|x_{n}-x_{n-1}||+\frac{1}{\mu}\left|r_{n-1}-r_{n}\right|\right\| u_{n-1}-x_{n-1} \| \\
& +\left|\beta_{n}-\beta_{n-1}\right|\left(\left\|S x_{n-1}\right\|+\left\|z_{n-1}\right\|\right) .
\end{aligned}
$$

Next, we estimate

$$
\begin{aligned}
\left\|x_{n+1}-x_{n}\right\| \leq & \| \alpha_{n} f\left(x_{n}\right)+\sum_{i=1}^{n}\left(\alpha_{i-1}-\alpha_{i}\right) V_{i} y_{n} \\
& -\left(\alpha_{n-1} f\left(x_{n-1}\right)+\sum_{i=1}^{n-1}\left(\alpha_{i-1}-\alpha_{i}\right) V_{i} y_{n-1}\right) \| \\
= & \| \alpha_{n}\left(f\left(x_{n}\right)-f\left(x_{n-1}\right)\right)+\left(\alpha_{n}-\alpha_{n-1}\right) f\left(x_{n-1}\right) \\
& +\sum_{i=1}^{n}\left(\alpha_{i-1}-\alpha_{i}\right)\left(V_{i} y_{n}-V_{i} y_{n-1}\right) \\
& +\left(\alpha_{n-1}-\alpha_{n}\right) V_{n} y_{n-1} \| \\
\leq & \alpha_{n}\left\|f\left(x_{n}\right)-f\left(x_{n-1}\right)\right\| \\
& +\sum_{i=1}^{n}\left(\alpha_{i-1}-\alpha_{i}\right)\left\|V_{i} y_{n}-V_{i} y_{n-1}\right\| \\
& +\left|\alpha_{n}-\alpha_{n-1}\right|\left(\left\|f\left(x_{n-1}\right)\right\|+\left\|V_{n} y_{n-1}\right\|\right) \\
\leq & \alpha_{n} \rho\left\|x_{n}-x_{n-1}\right\|+\sum_{i=1}^{n}\left(\alpha_{i-1}-\alpha_{i}\right)\left\|y_{n}-y_{n-1}\right\| \\
& +\left|\alpha_{n}-\alpha_{n-1}\right|\left(\left\|f\left(x_{n-1}\right)\right\|+\left\|V_{n} y_{n-1}\right\|\right) \\
= & \alpha_{n} \rho\left\|x_{n}-x_{n-1}\right\|+\left(1-\alpha_{n}\right)\left\|y_{n}-y_{n-1}\right\| \\
& +\left|\alpha_{n}-\alpha_{n-1}\right|\left(\left\|f\left(x_{n-1}\right)\right\|+\left\|V_{n} y_{n-1}\right\|\right) .
\end{aligned}
$$

From (46) and (47), we have

$$
\begin{aligned}
\left\|x_{n+1}-x_{n}\right\| \leq & \alpha_{n} \rho\left\|x_{n}-x_{n-1}\right\|+\left(1-\alpha_{n}\right) \\
& \times\left\{\left\|x_{n}-x_{n-1}\right\|+\frac{1}{\mu}\left|r_{n-1}-r_{n}\right|\left\|u_{n-1}-x_{n-1}\right\|\right. \\
& \left.\quad+\left|\beta_{n}-\beta_{n-1}\right|\left(\left\|S x_{n-1}\right\|+\left\|z_{n-1}\right\|\right)\right\} \\
& +\left|\alpha_{n}-\alpha_{n-1}\right|\left(\left\|f\left(x_{n-1}\right)\right\|+\left\|V_{n} y_{n-1}\right\|\right) \\
\leq & \left(1-(1-\rho) \alpha_{n}\right)\left\|x_{n}-x_{n-1}\right\|+\frac{1}{\mu}\left|r_{n-1}-r_{n}\right| \\
& \times\left\|u_{n-1}-x_{n-1}\right\| \\
& +\left|\beta_{n}-\beta_{n-1}\right|\left(\left\|S x_{n-1}\right\|+\left\|z_{n-1}\right\|\right)
\end{aligned}
$$

$$
\begin{aligned}
& +\left|\alpha_{n}-\alpha_{n-1}\right|\left(\left\|f\left(x_{n-1}\right)\right\|+\left\|V_{n} y_{n-1}\right\|\right) \\
\leq & \left(1-(1-\rho) \alpha_{n}\right)\left\|x_{n}-x_{n-1}\right\|+M \\
& \times\left(\frac{1}{\mu}\left|r_{n-1}-r_{n}\right|+\left|\beta_{n}-\beta_{n-1}\right|+\left|\alpha_{n}-\alpha_{n-1}\right|\right),
\end{aligned}
$$

where

$$
\begin{gathered}
M=\max \left\{\sup _{n \geq 1}\left\|u_{n-1}-x_{n-1}\right\|, \sup _{n \geq 1}\left(\left\|S x_{n-1}\right\|+\left\|z_{n-1}\right\|\right),\right. \\
\left.\sup _{n \geq 1}\left(\left\|f\left(x_{n-1}\right)\right\|+\left\|V_{n} y_{n-1}\right\|\right)\right\} .
\end{gathered}
$$

It follows by conditions (a)-(d) of Algorithm 11 and Lemma 7 that

$$
\lim _{n \rightarrow \infty}\left\|x_{n+1}-x_{n}\right\|=0 .
$$

Next, we show that $\lim _{n \rightarrow \infty}\left\|u_{n}-x_{n}\right\|=0$. Since $x^{*} \in S^{*} \cap$ $\operatorname{MEP}(F) \cap F(T)$ and $\alpha_{n}+\sum_{i=1}^{n}\left(\alpha_{i-1}-\alpha_{i}\right)=1$, by using (31) and (35), we obtain

$$
\begin{aligned}
\left\|x_{n+1}-x^{*}\right\|^{2} & \| \alpha_{n} f\left(x_{n}\right)+\sum_{i=1}^{n}\left(\alpha_{i-1}-\alpha_{i}\right) V_{i} y_{n}-\alpha_{n} x^{*} \\
& -\sum_{i=1}^{n}\left(\alpha_{i-1}-\alpha_{i}\right) V_{i} x^{*} \|^{2} \\
\leq & \alpha_{n}\left\|f\left(x_{n}\right)-x^{*}\right\|^{2}+\sum_{i=1}^{n}\left(\alpha_{i-1}-\alpha_{i}\right)\left\|V_{i} y_{n}-V_{i} x^{*}\right\|^{2} \\
\leq & \alpha_{n}\left\|f\left(x_{n}\right)-x^{*}\right\|^{2}+\sum_{i=1}^{n}\left(\alpha_{i-1}-\alpha_{i}\right)\left\|y_{n}-x^{*}\right\|^{2} \\
\leq & \alpha_{n}\left\|f\left(x_{n}\right)-x^{*}\right\|^{2}+\left(1-\alpha_{n}\right) \\
& \times\left(\beta_{n}\left\|S x_{n}-x^{*}\right\|^{2}+\left(1-\beta_{n}\right)\left\|z_{n}-x^{*}\right\|^{2}\right) \\
\leq & \alpha_{n}\left\|f\left(x_{n}\right)-x^{*}\right\|^{2}+\left(1-\alpha_{n}\right) \beta_{n}\left\|S x_{n}-x^{*}\right\|^{2} \\
& +\left(1-\alpha_{n}\right)\left(1-\beta_{n}\right) \\
& \times\left\{\left\|u_{n}-x^{*}\right\|^{2}-\mu_{2}\left(2 \theta_{2}-\mu_{2}\right)\right. \\
& \left.\times\left\|B_{2} u_{n}-B_{2} x^{*}\right\|^{2}-\mu_{1}\left(2 \theta_{1}-\mu_{1}\right)\left\|B_{1} v_{n}-B_{1} y^{*}\right\|^{2}\right\} \\
\leq & \alpha_{n}\left\|f\left(x_{n}\right)-x^{*}\right\|^{2}+\beta_{n}\left\|S x_{n}-x^{*}\right\|^{2} \\
+ & \left(1-\alpha_{n}\right)\left(1-\beta_{n}\right) \\
\times & \left\{\left\|x_{n}-x^{*}\right\|^{2}-r_{n}\left(2 \eta-r_{n}\right)\left\|D x_{n}-D x^{*}\right\|^{2}\right. \\
& \quad-\mu_{2}\left(2 \theta_{2}-\mu_{2}\right)\left\|B_{2} u_{n}-B_{2} x^{*}\right\|^{2} \\
& \left.-\mu_{1}\left(2 \theta_{1}-\mu_{1}\right)\left\|B_{1} v_{n}-B_{1} y^{*}\right\|^{2}\right\} \\
\leq &
\end{aligned}
$$




$$
\begin{aligned}
\leq \alpha_{n}\left\|f\left(x_{n}\right)-x^{*}\right\|^{2}+ & \beta_{n}\left\|S x_{n}-x^{*}\right\|^{2}+\left\|x_{n}-x^{*}\right\|^{2} \\
-\left(1-\alpha_{n}\right)\left(1-\beta_{n}\right) & \left\{r_{n}\left(2 \eta-r_{n}\right)\left\|D x_{n}-D x^{*}\right\|^{2}\right. \\
& +\mu_{2}\left(2 \theta_{2}-\mu_{2}\right)\left\|B_{2} u_{n}-B_{2} x^{*}\right\|^{2} \\
& \left.+\mu_{1}\left(2 \theta_{1}-\mu_{1}\right)\left\|B_{1} v_{n}-B_{1} y^{*}\right\|^{2}\right\} .
\end{aligned}
$$

Then, from the previous inequality, we get

$$
\begin{gathered}
\left(1-\alpha_{n}\right)\left(1-\beta_{n}\right)\left\{r_{n}\left(2 \eta-r_{n}\right)\left\|D x_{n}-D x^{*}\right\|^{2}\right. \\
+\mu_{2}\left(2 \theta_{2}-\mu_{2}\right)\left\|B_{2} u_{n}-B_{2} x^{*}\right\|^{2} \\
\left.\quad+\mu_{1}\left(2 \theta_{1}-\mu_{1}\right)\left\|B_{1} v_{n}-B_{1} y^{*}\right\|^{2}\right\} \\
\leq \alpha_{n}\left\|f\left(x_{n}\right)-x^{*}\right\|^{2}+\beta_{n}\left\|S x_{n}-x^{*}\right\|^{2} \\
+\left\|x_{n}-x^{*}\right\|^{2}-\left\|x_{n+1}-x^{*}\right\|^{2} \\
\leq \alpha_{n}\left\|f\left(x_{n}\right)-x^{*}\right\|^{2}+\beta_{n}\left\|S x_{n}-x^{*}\right\|^{2} \\
+\left(\left\|x_{n}-x^{*}\right\|+\left\|x_{n+1}-x^{*}\right\|\right)\left\|x_{n+1}-x_{n}\right\| .
\end{gathered}
$$

Since liminf $\operatorname{in}_{n \rightarrow \infty} r_{n} \leq \limsup _{n \rightarrow \infty} r_{n}<2 \eta, \lim _{n \rightarrow \infty} \| x_{n+1}-$ $x_{n} \|=0, \alpha_{n} \rightarrow 0$, and $\beta_{n} \rightarrow 0$, we obtain $\lim _{n \rightarrow \infty} \| B_{2} u_{n}-$ $B_{2} x^{*}\left\|=0, \lim _{n \rightarrow \infty}\right\| B_{1} v_{n}-B_{1} y^{*} \|=0$, and $\lim _{n \rightarrow \infty} \| D x_{n}-$ $D x^{*} \|=0$.

Since $T_{r_{n}}$ is firmly nonexpansive, we have

$$
\begin{aligned}
\left\|u_{n}-x^{*}\right\|^{2}= & \left\|T_{r_{n}}\left(x_{n}-r_{n} D x_{n}\right)-T_{r_{n}}\left(x^{*}-r_{n} D x^{*}\right)\right\|^{2} \\
\leq & \left\langle u_{n}-x^{*},\left(x_{n}-r_{n} D x_{n}\right)-\left(x^{*}-r_{n} D x^{*}\right)\right\rangle \\
= & \frac{1}{2}\left\{\left\|u_{n}-x^{*}\right\|^{2}\right. \\
+ & \left\|\left(x_{n}-r_{n} D x_{n}\right)-\left(x^{*}-r_{n} D x^{*}\right)\right\|^{2} \\
& -\| u_{n}-x^{*} \\
& \left.-\left[\left(x_{n}-r_{n} D x_{n}\right)-\left(x^{*}-r_{n} D x^{*}\right)\right] \|^{2}\right\} .
\end{aligned}
$$

Hence,

$$
\begin{aligned}
\left\|u_{n}-x^{*}\right\|^{2} \leq & \left\|\left(x_{n}-r_{n} D x_{n}\right)-\left(x^{*}-r_{n} D x^{*}\right)\right\|^{2} \\
& -\left\|u_{n}-x_{n}+r_{n}\left(D x_{n}-D x^{*}\right)\right\|^{2} \\
\leq & \left\|x_{n}-x^{*}\right\|^{2}-\left\|u_{n}-x_{n}+r_{n}\left(D x_{n}-D x^{*}\right)\right\|^{2} \\
\leq & \left\|x_{n}-x^{*}\right\|^{2}-\left\|u_{n}-x_{n}\right\|^{2} \\
& +2 r_{n}\left\|u_{n}-x_{n}\right\|\left\|D x_{n}-D x^{*}\right\| .
\end{aligned}
$$

From (51), (35), and the previous inequality, we have

$$
\begin{aligned}
\left\|x_{n+1}-x^{*}\right\|^{2} \leq & \alpha_{n}\left\|f\left(x_{n}\right)-x^{*}\right\|^{2} \\
+ & \left(1-\alpha_{n}\right)\left(\beta_{n}\left\|S x_{n}-x^{*}\right\|^{2}\right. \\
& \left.+\left(1-\beta_{n}\right)\left\|z_{n}-x^{*}\right\|^{2}\right) \\
\leq & \alpha_{n}\left\|f\left(x_{n}\right)-x^{*}\right\|^{2}+\left(1-\alpha_{n}\right) \\
& \times\left(\beta_{n}\left\|S x_{n}-x^{*}\right\|^{2}+\left(1-\beta_{n}\right)\left\|u_{n}-x^{*}\right\|^{2}\right) \\
\leq & \alpha_{n}\left\|f\left(x_{n}\right)-x^{*}\right\|^{2}+\left(1-\alpha_{n}\right) \\
& \times\left\{\beta_{n}\left\|S x_{n}-x^{*}\right\|^{2}+\left(1-\beta_{n}\right)\right. \\
& \times\left(\left\|x_{n}-x^{*}\right\|^{2}-\left\|u_{n}-x_{n}\right\|^{2}\right. \\
& \left.\left.+2 r_{n}\left\|u_{n}-x_{n}\right\|\left\|D x_{n}-D x^{*}\right\|\right)\right\} \\
\leq & \alpha_{n}\left\|f\left(x_{n}\right)-x^{*}\right\|^{2}+\beta_{n}\left\|S x_{n}-x^{*}\right\|^{2} \\
& +\left\|x_{n}-x^{*}\right\|^{2}-\left(1-\alpha_{n}\right)\left(1-\beta_{n}\right)\left\|u_{n}-x_{n}\right\|^{2} \\
& +2 r_{n}\left\|u_{n}-x_{n}\right\|\left\|D x_{n}-D x^{*}\right\| .
\end{aligned}
$$

Hence,

$$
\begin{aligned}
& \left(1-\alpha_{n}\right)\left(1-\beta_{n}\right)\left\|u_{n}-x_{n}\right\|^{2} \leq \alpha_{n}\left\|f\left(x_{n}\right)-x^{*}\right\|^{2} \\
& \quad+\beta_{n}\left\|S x_{n}-x^{*}\right\|^{2}+\left\|x_{n}-x^{*}\right\|^{2} \\
& \quad-\left\|x_{n+1}-x^{*}\right\|^{2} \\
& \quad+2 r_{n}\left\|u_{n}-x_{n}\right\|\left\|D x_{n}-D x^{*}\right\| \\
& \leq \alpha_{n}\left\|f\left(x_{n}\right)-x^{*}\right\|^{2}+\beta_{n}\left\|S x_{n}-x^{*}\right\|^{2} \\
& \quad+\left(\left\|x_{n}-x^{*}\right\|+\left\|x_{n+1}-x^{*}\right\|\right)\left\|x_{n+1}-x_{n}\right\| \\
& \quad+2 r_{n}\left\|u_{n}-x_{n}\right\|\left\|D x_{n}-D x^{*}\right\| .
\end{aligned}
$$

Since $\lim _{n \rightarrow \infty}\left\|x_{n+1}-x_{n}\right\|=0, \alpha_{n} \rightarrow 0, \beta_{n} \rightarrow 0$, and $\lim _{n \rightarrow \infty}\left\|D x_{n}-D x^{*}\right\|=0$, we obtain

$$
\lim _{n \rightarrow \infty}\left\|u_{n}-x_{n}\right\|=0
$$

From (21), we get

$$
\begin{aligned}
& \left\|v_{n}-y^{*}\right\|^{2} \\
& =\left\|P_{C}\left[u_{n}-\mu_{2} B_{2} u_{n}\right]-P_{C}\left[x^{*}-\mu_{2} B_{2} x^{*}\right]\right\|^{2} \\
& \leq\left\langle v_{n}-y^{*},\left(u_{n}-\mu_{2} B_{2} u_{n}\right)-\left(x^{*}-\mu_{2} B_{2} x^{*}\right)\right\rangle \\
& =\frac{1}{2}\left\{\left\|v_{n}-y^{*}\right\|^{2}+\left\|u_{n}-x^{*}-\mu_{2}\left(B_{2} u_{n}-B_{2} x^{*}\right)\right\|^{2}\right. \\
& \left.\quad \quad-\left\|u_{n}-x^{*}-\mu_{2}\left(B_{2} u_{n}-B_{2} x^{*}\right)-\left(v_{n}-y^{*}\right)\right\|^{2}\right\}
\end{aligned}
$$




$$
\begin{aligned}
& \leq \frac{1}{2}\left\{\left\|v_{n}-y^{*}\right\|^{2}+\left\|u_{n}-x^{*}\right\|^{2}\right. \\
& -\mu_{2}\left(2 \theta_{2}-\mu_{2}\right)\left\|B_{2} u_{n}-B_{2} x^{*}\right\|^{2} \\
& -\| u_{n}-x^{*}-\mu_{2}\left(B_{2} u_{n}-B_{2} x^{*}\right) \\
& \left.\quad-\left(v_{n}-y^{*}\right) \|^{2}\right\} \\
& \leq \frac{1}{2}\left\{\left\|v_{n}-y^{*}\right\|^{2}+\left\|u_{n}-x^{*}\right\|^{2}\right. \\
& \left.-\left\|u_{n}-v_{n}-\mu_{2}\left(B_{2} u_{n}-B_{2} x^{*}\right)-\left(x^{*}-y^{*}\right)\right\|^{2}\right\} \\
& \leq \frac{1}{2}\left\{\left\|v_{n}-y^{*}\right\|^{2}+\left\|u_{n}-x^{*}\right\|^{2}\right. \\
& -\left\|u_{n}-v_{n}-\left(x^{*}-y^{*}\right)\right\|^{2} \\
& +2 \mu_{2}\left\langle u_{n}-v_{n}-\left(x^{*}-y^{*}\right), B_{2} u_{n}-B_{2} x^{*}\right\rangle \\
& \left.-\mu_{2}^{2}\left\|B_{2} u_{n}-B_{2} x^{*}\right\|^{2}\right\} \\
& \leq \frac{1}{2}\left\{\left\|v_{n}-y^{*}\right\|^{2}+\left\|u_{n}-x^{*}\right\|^{2}\right. \\
& \quad-\left\|u_{n}-v_{n}-\left(x^{*}-y^{*}\right)\right\|^{2} \\
& \left.+2 \mu_{2}\left\|u_{n}-v_{n}-\left(x^{*}-y^{*}\right)\right\|\left\|B_{2} u_{n}-B_{2} x^{*}\right\|\right\} .
\end{aligned}
$$

Hence,

$$
\begin{aligned}
\left\|v_{n}-y^{*}\right\|^{2} \leq & \left\|u_{n}-x^{*}\right\|^{2}-\left\|u_{n}-v_{n}-\left(x^{*}-y^{*}\right)\right\|^{2} \\
& +2 \mu_{2}\left\|u_{n}-v_{n}-\left(x^{*}-y^{*}\right)\right\|\left\|B_{2} u_{n}-B_{2} x^{*}\right\| \\
\leq & \left\|x_{n}-x^{*}\right\|^{2}-\left\|u_{n}-x_{n}\right\|^{2} \\
& +2 r_{n}\left\|u_{n}-x_{n}\right\|\left\|D x_{n}-D x^{*}\right\| \\
& -\left\|u_{n}-v_{n}-\left(x^{*}-y^{*}\right)\right\|^{2} \\
& +2 \mu_{2}\left\|u_{n}-v_{n}-\left(x^{*}-y^{*}\right)\right\|\left\|B_{2} u_{n}-B_{2} x^{*}\right\|,
\end{aligned}
$$

where the last inequality follows from (54). On the other hand, from (29) and (21), we obtain

$$
\begin{gathered}
\left\|z_{n}-x^{*}\right\|^{2}=\left\|P_{C}\left[v_{n}-\mu_{1} B_{1} v_{n}\right]-P_{C}\left[y^{*}-\mu_{1} B_{1} y^{*}\right]\right\|^{2} \\
\leq\left\langle z_{n}-x^{*},\left(v_{n}-\mu_{1} B_{1} v_{n}\right)-\left(y^{*}-\mu_{1} B_{1} y^{*}\right)\right\rangle \\
=\frac{1}{2}\left\{\left\|z_{n}-x^{*}\right\|^{2}+\left\|v_{n}-y^{*}-\mu_{1}\left(B_{1} v_{n}-B_{1} y^{*}\right)\right\|^{2}\right. \\
\quad-\| v_{n}-y^{*}-\mu_{1}\left(B_{1} v_{n}-B_{1} y^{*}\right) \\
\left.\quad-\left(z_{n}-x^{*}\right) \|^{2}\right\} \\
=\frac{1}{2}\left\{\left\|z_{n}-x^{*}\right\|^{2}+\left\|v_{n}-y^{*}\right\|^{2}\right. \\
\quad-2 \mu_{1}\left\langle v_{n}-y^{*}, B_{1} v_{n}-B_{1} y^{*}\right\rangle
\end{gathered}
$$

$$
\begin{gathered}
+\mu_{1}^{2}\left\|B_{1} v_{n}-B_{1} y^{*}\right\|^{2} \\
-\| v_{n}-y^{*}-\mu_{1}\left(B_{1} v_{n}-B_{1} y^{*}\right) \\
\left.-\left(z_{n}-x^{*}\right) \|^{2}\right\} \\
\leq \frac{1}{2}\left\{\left\|z_{n}-x^{*}\right\|^{2}+\left\|v_{n}-y^{*}\right\|^{2}\right. \\
-\mu_{1}\left(2 \theta_{1}-\mu_{1}\right)\left\|B_{1} v_{n}-B_{1} y^{*}\right\|^{2} \\
-\| v_{n}-y^{*}-\mu_{1}\left(B_{1} v_{n}-B_{1} y^{*}\right) \\
\left.-\left(z_{n}-x^{*}\right) \|^{2}\right\} \\
\leq \frac{1}{2}\left\{\left\|z_{n}-x^{*}\right\|^{2}+\left\|v_{n}-y^{*}\right\|^{2}\right. \\
-\| v_{n}-z_{n}-\mu_{1}\left(B_{1} v_{n}-B_{1} y^{*}\right) \\
\left.+\left(x^{*}-y^{*}\right) \|^{2}\right\} \\
\leq \frac{1}{2}\left\{\left\|z_{n}-x^{*}\right\|^{2}+\left\|v_{n}-y^{*}\right\|^{2}\right. \\
-\left\|v_{n}-z_{n}+\left(x^{*}-y^{*}\right)\right\|^{2} \\
\left.+2 \mu_{1}\left\langle v_{n}-z_{n}+\left(x^{*}-y^{*}\right), B_{1} v_{n}-B_{1} y^{*}\right\rangle\right\} \\
\leq \frac{1}{2}\left\{\left\|z_{n}-x^{*}\right\|^{2}+\left\|v_{n}-y^{*}\right\|^{2}\right. \\
-\left\|v_{n}-z_{n}+\left(x^{*}-y^{*}\right)\right\|^{2} \\
+2 \mu_{1}\left\|v_{n}-z_{n}+\left(x^{*}-y^{*}\right)\right\| \\
\left.\times\left\|B_{1} v_{n}-B_{1} y^{*}\right\|\right\} .
\end{gathered}
$$

Hence,

$$
\begin{aligned}
\left\|z_{n}-x^{*}\right\|^{2} \leq & \left\|v_{n}-y^{*}\right\|^{2}-\left\|v_{n}-z_{n}+\left(x^{*}-y^{*}\right)\right\|^{2} \\
& +2 \mu_{1}\left\|v_{n}-z_{n}+\left(x^{*}-y^{*}\right)\right\|\left\|B_{1} v_{n}-B_{1} y^{*}\right\| \\
\leq & \left\|x_{n}-x^{*}\right\|^{2}-\left\|u_{n}-x_{n}\right\|^{2} \\
& +2 r_{n}\left\|u_{n}-x_{n}\right\|\left\|D x_{n}-D x^{*}\right\| \\
& -\left\|u_{n}-v_{n}-\left(x^{*}-y^{*}\right)\right\|^{2} \\
& +2 \mu_{2}\left\|u_{n}-v_{n}-\left(x^{*}-y^{*}\right)\right\|\left\|B_{2} u_{n}-B_{2} x^{*}\right\| \\
& -\left\|v_{n}-z_{n}+\left(x^{*}-y^{*}\right)\right\|^{2} \\
& +2 \mu_{1}\left\|v_{n}-z_{n}+\left(x^{*}-y^{*}\right)\right\|\left\|B_{1} v_{n}-B_{1} y^{*}\right\|,
\end{aligned}
$$

where the last inequality follows from (59). From (51) and the previous inequality, we have

$$
\begin{aligned}
\left\|x_{n+1}-x^{*}\right\|^{2} \leq & \alpha_{n}\left\|f\left(x_{n}\right)-x^{*}\right\|^{2}+\left(1-\alpha_{n}\right) \\
& \times\left(\beta_{n}\left\|S x_{n}-x^{*}\right\|^{2}+\left(1-\beta_{n}\right)\left\|z_{n}-x^{*}\right\|^{2}\right)
\end{aligned}
$$




$$
\begin{gathered}
\leq \alpha_{n}\left\|f\left(x_{n}\right)-x^{*}\right\|^{2}+\left(1-\alpha_{n}\right) \\
\times\left\{\beta_{n}\left\|S x_{n}-x^{*}\right\|^{2}\right. \\
+\left(1-\beta_{n}\right)\left(\left\|x_{n}-x^{*}\right\|^{2}-\left\|u_{n}-x_{n}\right\|^{2}\right. \\
\left.+2 r_{n}\left\|u_{n}-x_{n}\right\|\left\|D x_{n}-D x^{*}\right\|\right) \\
+\left(1-\beta_{n}\right)\left(-\left\|u_{n}-v_{n}-\left(x^{*}-y^{*}\right)\right\|^{2}\right. \\
+2 \mu_{2}\left\|u_{n}-v_{n}-\left(x^{*}-y^{*}\right)\right\| \\
\left.\times\left\|B_{2} u_{n}-B_{2} x^{*}\right\|\right) \\
+\left(1-\beta_{n}\right)\left(-\left\|v_{n}-z_{n}+\left(x^{*}-y^{*}\right)\right\|^{2}\right. \\
+2 \mu_{1}\left\|v_{n}-z_{n}+\left(x^{*}-y^{*}\right)\right\| \\
\left.\left.\times\left\|B_{1} v_{n}-B_{1} y^{*}\right\|\right)\right\} \\
\leq \alpha_{n}\left\|f\left(x_{n}\right)-x^{*}\right\|^{2}+\beta_{n}\left\|S x_{n}-x^{*}\right\|^{2} \\
+\left\|x_{n}-x^{*}\right\|^{2}+2 r_{n}\left\|u_{n}-x_{n}\right\| \\
\times\left\|D x_{n}-D x^{*}\right\| \\
+2 \mu_{2}\left\|u_{n}-v_{n}-\left(x^{*}-y^{*}\right)\right\| \\
\times\left\|B_{2} u_{n}-B_{2} x^{*}\right\|+2 \mu_{1}\left\|v_{n}-z_{n}+\left(x^{*}-y^{*}\right)\right\| \\
\times\left\|B_{1} v_{n}-B_{1} y^{*}\right\|-\left(1-\alpha_{n}\right)\left(1-\beta_{n}\right) \\
\times\left\{\left\|u_{n}-x_{n}\right\|^{2}+\left\|u_{n}-v_{n}-\left(x^{*}-y^{*}\right)\right\|^{2}\right. \\
\left.+\left\|v_{n}-z_{n}+\left(x^{*}-y^{*}\right)\right\|^{2}\right\} .
\end{gathered}
$$

Hence,

$$
\begin{aligned}
&\left(1-\alpha_{n}\right)\left(1-\beta_{n}\right) \\
& \times\left\{\left\|u_{n}-x_{n}\right\|^{2}+\left\|u_{n}-v_{n}-\left(x^{*}-y^{*}\right)\right\|^{2}\right. \\
&\left.+\left\|v_{n}-z_{n}+\left(x^{*}-y^{*}\right)\right\|^{2}\right\} \\
& \leq \alpha_{n}\left\|f\left(x_{n}\right)-x^{*}\right\|^{2}+\beta_{n}\left\|S x_{n}-x^{*}\right\|^{2} \\
&+\left\|x_{n}-x^{*}\right\|^{2}-\left\|x_{n+1}-x^{*}\right\|^{2} \\
&+2 r_{n}\left\|u_{n}-x_{n}\right\|\left\|D x_{n}-D x^{*}\right\| \\
&+2 \mu_{2}\left\|u_{n}-v_{n}-\left(x^{*}-y^{*}\right)\right\|\left\|B_{2} u_{n}-B_{2} x^{*}\right\| \\
&+2 \mu_{1}\left\|v_{n}-z_{n}+\left(x^{*}-y^{*}\right)\right\|\left\|B_{1} v_{n}-B_{1} y^{*}\right\| \\
& \leq \alpha_{n}\left\|f\left(x_{n}\right)-x^{*}\right\|^{2}+\beta_{n}\left\|S x_{n}-x^{*}\right\|^{2} \\
&+\left(\left\|x_{n}-x^{*}\right\|+\left\|x_{n+1}-x^{*}\right\|\right)\left\|x_{n+1}-x_{n}\right\| \\
&+2 r_{n}\left\|u_{n}-x_{n}\right\|\left\|D x_{n}-D x^{*}\right\|
\end{aligned}
$$

$$
\begin{aligned}
& +2 \mu_{2}\left\|u_{n}-v_{n}-\left(x^{*}-y^{*}\right)\right\|\left\|B_{2} u_{n}-B_{2} x^{*}\right\| \\
& +2 \mu_{1}\left\|v_{n}-z_{n}+\left(x^{*}-y^{*}\right)\right\|\left\|B_{1} v_{n}-B_{1} y^{*}\right\| .
\end{aligned}
$$

Since $\lim _{n \rightarrow \infty}\left\|x_{n+1}-x_{n}\right\|=0, \alpha_{n} \rightarrow 0, \beta_{n} \rightarrow 0$, and $\lim _{n \rightarrow \infty}\left\|D x_{n}-D x^{*}\right\|=0, \lim _{n \rightarrow \infty}\left\|B_{2} u_{n}-B_{2} x^{*}\right\|=0$, $\lim _{n \rightarrow \infty}\left\|B_{1} v_{n}-B_{1} y^{*}\right\|=0$, we obtain

$$
\begin{aligned}
& \lim _{n \rightarrow \infty}\left\|u_{n}-v_{n}-\left(x^{*}-y^{*}\right)\right\|=0, \\
& \lim _{n \rightarrow \infty}\left\|v_{n}-z_{n}+\left(x^{*}-y^{*}\right)\right\|=0 .
\end{aligned}
$$

Since

$$
\begin{aligned}
\left\|u_{n}-z_{n}\right\| \leq & \left\|u_{n}-v_{n}-\left(x^{*}-y^{*}\right)\right\| \\
& +\left\|v_{n}-z_{n}+\left(x^{*}-y^{*}\right)\right\|,
\end{aligned}
$$

we get

$$
\lim _{n \rightarrow \infty}\left\|u_{n}-z_{n}\right\|=0
$$

It follows from (57) and (66) that

$$
\lim _{n \rightarrow \infty}\left\|x_{n}-z_{n}\right\|=0
$$

Now, let $z \in F(T) \cap S^{*} \cap \operatorname{MEP}(F)$; since for each $i \geq 1, V_{i} x_{n} \in$ $C$, and $\alpha_{n}+\sum_{i=1}^{n}\left(\alpha_{i-1}-\alpha_{i}\right)=1$, we have $\sum_{i=1}^{n}\left(\alpha_{i-1}-\alpha_{i}\right) V_{i} x_{n}+$ $\alpha_{n} z \in C$. And

$$
\begin{aligned}
\sum_{i=1}^{n}\left(\alpha_{i-1}-\alpha_{i}\right)\left(x_{n}-V_{i} x_{n}\right) & \\
= & P_{C}\left[\alpha_{n} f\left(x_{n}\right)+\sum_{i=1}^{n}\left(\alpha_{i-1}-\alpha_{i}\right) V_{i} y_{n}\right]+\left(1-\alpha_{n}\right) x_{n} \\
& -\left(\sum_{i=1}^{n}\left(\alpha_{i-1}-\alpha_{i}\right) V_{i} x_{n}+\alpha_{n} z\right)+\alpha_{n} z-x_{n+1} \\
= & P_{C}\left[\alpha_{n} f\left(x_{n}\right)+\sum_{i=1}^{n}\left(\alpha_{i-1}-\alpha_{i}\right) V_{i} y_{n}\right]+\alpha_{n}\left(z-x_{n+1}\right) \\
& -P_{C}\left[\sum_{i=1}^{n}\left(\alpha_{i-1}-\alpha_{i}\right) V_{i} x_{n}+\alpha_{n} z\right] \\
& +\left(1-\alpha_{n}\right)\left(x_{n}-x_{n+1}\right) .
\end{aligned}
$$


It follows that

$$
\begin{aligned}
& \sum_{i=1}^{n}\left(\alpha_{i-1}-\alpha_{i}\right)\left\langle x_{n}-V_{i} x_{n}, x_{n}-x^{*}\right\rangle \\
&=\left\langle P_{C}\left[\alpha_{n} f\left(x_{n}\right)+\sum_{i=1}^{n}\left(\alpha_{i-1}-\alpha_{i}\right) V_{i} y_{n}\right]\right. \\
&\left.\quad-P_{C}\left[\sum_{i=1}^{n}\left(\alpha_{i-1}-\alpha_{i}\right) V_{i} x_{n}+\alpha_{n} z\right], x_{n}-x^{*}\right\rangle \\
& \quad+\alpha_{n}\left\langle z-x_{n+1}, x_{n}-x^{*}\right\rangle \\
& \quad+\left(1-\alpha_{n}\right)\left\langle x_{n}-x_{n+1}, x_{n}-x^{*}\right\rangle \\
& \leq\left\|\alpha_{n}\left(f\left(x_{n}\right)-z\right)+\sum_{i=1}^{n}\left(\alpha_{i-1}-\alpha_{i}\right)\left(V_{i} y_{n}-V_{i} x_{n}\right)\right\| \\
&+\left\|x_{n}-x^{*}\right\|+\alpha_{n}\left\|z-x_{n+1}\right\|\left\|x_{n}-x^{*}\right\| \\
&+\left(1-\alpha_{n}\right)\left\|x_{n}-x_{n+1}\right\|\left\|x_{n}-x^{*}\right\| \\
& \leq \alpha_{n}\left\|f\left(x_{n}\right)-z\right\|\left\|x_{n}-x^{*}\right\| \\
&+\sum_{i=1}^{n}\left(\alpha_{i-1}-\alpha_{i}\right)\left\|y_{n}-x_{n}\right\|\left\|x_{n}-x^{*}\right\| \\
&+\left(1-\alpha_{n}\right)\left\|x_{n}-x_{n+1}\right\|\left\|x_{n}-x^{*}\right\| . \\
&+\alpha_{n}\left\|f\left(x_{n}\right)-z\right\|\left\|x_{n}-x^{*}\right\| \\
&+\left(1-\alpha_{n}\right) \beta_{n}\left\|S x_{n}-x_{n}\right\|\left\|x_{n}-x^{*}\right\| \\
&+\alpha_{n}\left\|z-x_{n+1}\right\|\left\|x_{n}-x^{*}\right\| \\
&+\left(1-\alpha_{n}\right)\left\|x_{n}-x_{n+1}\right\|\left\|x_{n}-x_{n}-x_{n}\right\|\left\|x_{n}-x^{*}\right\| \\
&= \alpha_{n}\left\|f\left(x_{n}\right)-z\right\|\left\|x_{n}-x^{*}\right\| \\
&+\left(1-\alpha_{n}\right)\left\|y_{n}-x_{n}\right\|\left\|x_{n}-x^{*}\right\| \\
&+\left(1-\alpha_{n}\right)\left\|\alpha_{n} S-x_{n+1}\right\|\left\|x_{n}-x^{*}\right\| \\
&+\left(1-\alpha_{n}\right)\left\|x_{n}-x_{n+1}\right\|\left\|x_{n}-x^{*}\right\| \\
&+\left(1-x_{n}\right)\left\|x_{n}-x_{n+1}\right\|\left\|x_{n}-x_{n}^{*}\right\| \\
&+x_{n}-x_{n} \| \\
&+x_{n} \| \\
&+x_{n+1}\|\| x_{n}-x^{*} \| \\
&+(6)
\end{aligned}
$$

From Lemma 10 and the previous inequality, we get

$$
\frac{1}{2} \sum_{i=1}^{n}\left(\alpha_{i-1}-\alpha_{i}\right)\left\|x_{n}-V_{i} x_{n}\right\|^{2}
$$

$$
\begin{aligned}
\leq & \sum_{i=1}^{n}\left(\alpha_{i-1}-\alpha_{i}\right)\left\langle x_{n}-V_{i} x_{n}, x_{n}-x^{*}\right\rangle \\
\leq & \alpha_{n}\left\|f\left(x_{n}\right)-z\right\|\left\|x_{n}-x^{*}\right\| \\
& +\left(1-\alpha_{n}\right) \beta_{n}\left\|S x_{n}-x_{n}\right\|\left\|x_{n}-x^{*}\right\| \\
& +\left(1-\alpha_{n}\right)\left(1-\beta_{n}\right)\left\|z_{n}-x_{n}\right\|\left\|x_{n}-x^{*}\right\| \\
& +\alpha_{n}\left\|z-x_{n+1}\right\|\left\|x_{n}-x^{*}\right\| \\
& +\left(1-\alpha_{n}\right)\left\|x_{n}-x_{n+1}\right\|\left\|x_{n}-x^{*}\right\| .
\end{aligned}
$$

Since $\lim _{n \rightarrow \infty}\left\|x_{n+1}-x_{n}\right\|=0, \alpha_{n} \rightarrow 0, \beta_{n} \rightarrow 0$, and $\lim _{n \rightarrow \infty}\left\|x_{n}-z_{n}\right\|=0$, we obtain

$$
\lim _{n \rightarrow \infty} \sum_{i=1}^{n}\left(\alpha_{i-1}-\alpha_{i}\right)\left\|x_{n}-V_{i} x_{n}\right\|^{2}=0 .
$$

Since $\left(\alpha_{i-1}-\alpha_{i}\right)\left\|x_{n}-V_{i} x_{n}\right\|^{2} \leq \sum_{i=1}^{n}\left(\alpha_{i-1}-\alpha_{i}\right)\left\|x_{n}-V_{i} x_{n}\right\|^{2}$ and $\left\{\alpha_{n}\right\}$ is strictly decreasing, we have

$$
\lim _{n \rightarrow \infty}\left\|x_{n}-V_{i} x_{n}\right\|=0 .
$$

Hence, we obtain

$$
\lim _{n \rightarrow \infty}\left\|x_{n}-T_{i} x_{n}\right\|=\lim _{n \rightarrow \infty} \frac{\left\|x_{n}-V_{i} x_{n}\right\|}{\left(1-k_{i}\right)}=0, \quad \forall i \geq 1 .
$$

Since $\left\{x_{n}\right\}$ is bounded, without loss of generality, we can assume that $x_{n} \rightarrow w \in C$. It follows from Lemma 6 that $w \in F(T)$. Therefore, $w_{w}\left(x_{n}\right) \subset F(T)$.

Theorem 14. The sequence $\left\{x_{n}\right\}$ generated by Algorithm 11 converges strongly to $z=P_{S^{*} \cap \operatorname{MEP}(F) \cap F(T)} f(z)$, which is the unique solution of the variational inequality

$$
\langle(I-f) z, x-z\rangle \geq 0, \quad \forall x \in S^{*} \cap \operatorname{MEP}(F) \cap F(T) .
$$

Proof. Since $\left\{x_{n}\right\}$ is bounded $x_{n} \rightarrow w$ and from Lemma 13, we have $w \in F(T)$. Next, we show that $w \in \operatorname{MEP}(F)$. Since $u_{n}=T_{r_{n}}\left(x_{n}-r_{n} D x_{n}\right)$, we have

$$
F\left(u_{n}, y\right)+\left\langle D x_{n}, y-u_{n}\right\rangle+\frac{1}{r_{n}}\left\langle y-u_{n}, u_{n}-x_{n}\right\rangle \geq 0,
$$

$\forall y \in C$.

It follows from monotonicity of $F$ that

$$
\begin{array}{r}
\left\langle D x_{n}, y-u_{n}\right\rangle+\frac{1}{r_{n}}\left\langle y-u_{n}, u_{n}-x_{n}\right\rangle \geq F\left(y, u_{n}\right), \\
\forall y \in C,
\end{array}
$$

$$
\left\langle D x_{n_{k}}, y-u_{n_{k}}\right\rangle+\left\langle y-u_{n_{k}}, \frac{u_{n_{k}}-x_{n_{k}}}{r_{n_{k}}}\right\rangle \geq F\left(y, u_{n_{k}}\right),
$$

$\forall y \in C$. 
Since $\lim _{n \rightarrow \infty}\left\|u_{n}-x_{n}\right\|=0$ and $x_{n} \rightarrow w$, it easy to observe that $u_{n_{k}} \rightarrow w$. For any $0<t \leq 1$ and $y \in C$, let $y_{t}=t y+(1-$ $t) w$; we have $y_{t} \in C$. Then from (76), we obtain

$$
\begin{aligned}
\left\langle D y_{t}, y_{t}-u_{n_{k}}\right\rangle \geq & \left\langle D y_{t}, y_{t}-u_{n_{k}}\right\rangle-\left\langle D x_{n_{k}}, y_{t}-u_{n_{k}}\right\rangle \\
& -\left\langle y_{t}-u_{n_{k}}, \frac{u_{n_{k}}-x_{n_{k}}}{r_{n_{k}}}\right\rangle+F\left(y_{t}, u_{n_{k}}\right) \\
= & \left\langle D y_{t}-D u_{n_{k}}, y_{t}-u_{n_{k}}\right\rangle \\
& +\left\langle D u_{n_{k}}-D x_{n_{k}}, y_{t}-u_{n_{k}}\right\rangle \\
& -\left\langle y_{t}-u_{n_{k}}, \frac{u_{n_{k}}-x_{n_{k}}}{r_{n_{k}}}\right\rangle+F\left(y_{t}, u_{n_{k}}\right) .
\end{aligned}
$$

Since $D$ is Lipschitz continuous and $\lim _{n \rightarrow \infty}\left\|u_{n}-x_{n}\right\|=0$, we obtain $\lim _{k \rightarrow \infty}\left\|D u_{n_{k}}-D x_{n_{k}}\right\|=0$. From the monotonicity of $D$ and $u_{n_{k}} \rightarrow w$, it follows from (77) that

$$
\left\langle D y_{t}, y_{t}-w\right\rangle \geq F\left(y_{t}, w\right)
$$

Hence, from assumptions (i)-(iv) of Lemma 4 and (78), we have

$$
\begin{aligned}
0 & =F\left(y_{t}, y_{t}\right) \leq t F\left(y_{t}, y\right)+(1-t) F\left(y_{t}, w\right) \\
& \leq t F\left(y_{t}, y\right)+(1-t)\left\langle D y_{t}, y_{t}-w\right\rangle \\
& \leq t F\left(y_{t}, y\right)+(1-t) t\left\langle D y_{t}, y-w\right\rangle
\end{aligned}
$$

which implies that $F\left(y_{t}, y\right)+(1-t)\left\langle D y_{t}, y-w\right\rangle \geq 0$. Letting $t \rightarrow 0_{+}$, we have

$$
F(w, y)+\langle D w, y-w\rangle \geq 0, \quad \forall y \in C,
$$

which implies that $w \in \operatorname{MEP}(F)$. Next, we show that $w \in S^{*}$. Since $\lim _{n \rightarrow \infty}\left\|x_{n}-z_{n}\right\|=0$ and there exists a subsequence $\left\{x_{n_{k}}\right\}$ of $\left\{x_{n}\right\}$ such that $x_{n_{k}} \rightarrow w$, it easy to observe that $z_{n_{k}} \rightarrow$ $w$. For any $x, y \in C$, using (23), we have

$$
\begin{aligned}
& \|Q(x)-Q(y)\|^{2} \\
& =\| P_{C}\left[P_{C}\left[x-\mu_{2} B_{2} x\right]-\mu_{1} B_{1} P_{C}\left[x-\mu_{2} B_{2} x\right]\right] \\
& \quad-P_{C}\left[P_{C}\left[y-\mu_{2} B_{2} y\right]-\mu_{1} B_{1} P_{C}\left[y-\mu_{2} B_{2} y\right]\right] \|^{2} \\
& \leq \|\left(P_{C}\left[x-\mu_{2} B_{2} x\right]-P_{C}\left[y-\mu_{2} B_{2} y\right]\right) \\
& \quad-\mu_{1}\left(B_{1} P_{C}\left[x-\mu_{2} B_{2} x\right]-B_{1} P_{C}\left[y-\mu_{2} B_{2} y\right]\right) \|^{2}
\end{aligned}
$$

$$
\begin{aligned}
\leq & \left\|P_{C}\left[x-\mu_{2} B_{2} x\right]-P_{C}\left[y-\mu_{2} B_{2} y\right]\right\|^{2} \\
& -\mu_{1}\left(2 \theta_{1}-\mu_{1}\right)\left\|P_{C}\left[x-\mu_{2} B_{2} x\right]-P_{C}\left[y-\mu_{2} B_{2} y\right]\right\|^{2} \\
\leq & \left\|P_{C}\left[x-\mu_{2} B_{2} x\right]-P_{C}\left[y-\mu_{2} B_{2} y\right]\right\|^{2} \\
\leq & \left\|\left(x-\mu_{2} B_{2} x\right)-\left(y-\mu_{2} B_{2} y\right)\right\|^{2} \\
\leq & \|x-y\|^{2} \\
& -\mu_{2}\left(2 \theta_{2}-\mu_{2}\right)\left\|B_{2} x-B_{2} y\right\|^{2} \\
\leq & \|x-y\|^{2} .
\end{aligned}
$$

This implies that $Q: C \rightarrow C$ is nonexpansive. On the other hand,

$$
\begin{aligned}
\left\|z_{n}-Q\left(z_{n}\right)\right\|^{2} \\
=\| P_{C}\left[P_{C}\left[u_{n}-\mu_{2} B_{2} u_{n}\right]-\mu_{1} B_{1} P_{C}\left[u_{n}-\mu_{2} B_{2} u_{n}\right]\right] \\
\quad-Q\left(z_{n}\right) \|^{2} \\
=\left\|Q\left(u_{n}\right)-Q\left(z_{n}\right)\right\|^{2} \\
\leq\left\|u_{n}-z_{n}\right\|^{2} .
\end{aligned}
$$

Since $\lim _{n \rightarrow \infty}\left\|u_{n}-z_{n}\right\|=0$ (see (66)), we have $\lim _{n \rightarrow \infty} \| z_{n}-$ $Q\left(z_{n}\right) \|=0$. It follows from Lemma 6 that $w=Q(w)$, which implies from Lemma 3 that $w \in S^{*}$.

Thus, we have

$$
w \in S^{*} \cap \operatorname{MEP}(F) \cap F(T) .
$$

Next, we claim that $\lim _{\sup _{n \rightarrow \infty}}\left\langle f(z)-z, x_{n}-z\right\rangle \leq 0$ where $z=P_{S^{*} \cap M E P(F) \cap F(T)} f(z)$.

Since $\left\{x_{n}\right\}$ is bounded, there exists a subsequence $\left\{x_{n_{k}}\right\}$ of $\left\{x_{n}\right\}$ such that

$$
\begin{aligned}
\limsup _{n \rightarrow \infty}\left\langle f(z)-z, x_{n}-z\right\rangle & =\limsup _{k \rightarrow \infty}\left\langle f(z)-z, x_{n_{k}}-z\right\rangle \\
& =\langle f(z)-z, w-z\rangle \leq 0 .
\end{aligned}
$$

Next, we show that $x_{n} \rightarrow z$.

One has

$$
\begin{aligned}
\left\|x_{n+1}-z\right\|^{2}= & \left\langle x_{n+1}-\alpha_{n} f\left(x_{n}\right)\right. \\
& \left.-\sum_{i=1}^{n}\left(\alpha_{i-1}-\alpha_{i}\right) V_{i} y_{n}, x_{n+1}-z\right\rangle \\
& +\left\langle\alpha_{n} f\left(x_{n}\right)\right. \\
& \left.+\sum_{i=1}^{n}\left(\alpha_{i-1}-\alpha_{i}\right) V_{i} y_{n}-z, x_{n+1}-z\right\rangle
\end{aligned}
$$




$$
\begin{aligned}
& \leq\left\langle\alpha_{n} f\left(x_{n}\right)\right. \\
& \left.+\sum_{i=1}^{n}\left(\alpha_{i-1}-\alpha_{i}\right) V_{i} y_{n}-z, x_{n+1}-z\right\rangle \\
& =\alpha_{n}\left\langle f\left(x_{n}\right)-f(z), x_{n+1}-z\right\rangle \\
& +\alpha_{n}\left\langle f(z)-z, x_{n+1}-z\right\rangle \\
& +\sum_{i=1}^{n}\left(\alpha_{i-1}-\alpha_{i}\right)\left\langle V_{i} y_{n}-z, x_{n+1}-z\right\rangle \\
& \leq \alpha_{n}\left\|f\left(x_{n}\right)-f(z)\right\|\left\|x_{n+1}-z\right\| \\
& +\alpha_{n}\left\langle f(z)-z, x_{n+1}-z\right\rangle \\
& +\sum_{i=1}^{n}\left(\alpha_{i-1}-\alpha_{i}\right)\left\|V_{i} y_{n}-z\right\|\left\|x_{n+1}-z\right\| \\
& \leq \alpha_{n} \rho\left\|x_{n}-z\right\|\left\|x_{n+1}-z\right\| \\
& +\alpha_{n}\left\langle f(z)-z, x_{n+1}-z\right\rangle \\
& +\sum_{i=1}^{n}\left(\alpha_{i-1}-\alpha_{i}\right)\left\|y_{n}-z\right\|\left\|x_{n+1}-z\right\| \\
& \leq \alpha_{n} \rho\left\|x_{n}-z\right\|\left\|x_{n+1}-z\right\| \\
& +\alpha_{n}\left\langle f(z)-z, x_{n+1}-z\right\rangle+\left(1-\alpha_{n}\right) \\
& \times\left\{\beta_{n}\left\|S x_{n}-S z\right\|\right. \\
& \left.+\beta_{n}\|S z-z\|+\left(1-\beta_{n}\right)\left\|z_{n}-z\right\|\right\} \\
& \times\left\|x_{n+1}-z\right\| \\
& \leq \alpha_{n} \rho\left\|x_{n}-z\right\|\left\|x_{n+1}-z\right\| \\
& +\alpha_{n}\left\langle f(z)-z, x_{n+1}-z\right\rangle \\
& \times\left(1-\alpha_{n}\right)\left\{\beta_{n}\left\|x_{n}-z\right\|+\beta_{n}\|S z-z\|\right. \\
& \left.+\left(1-\beta_{n}\right)\left\|x_{n}-z\right\|\right\}\left\|x_{n+1}-z\right\| \\
& \leq\left(1-\alpha_{n}(1-\rho)\right)\left\|x_{n}-z\right\| \\
& \times\left\|x_{n+1}-z\right\|+\alpha_{n}\left\langle f(z)-z, x_{n+1}-z\right\rangle \\
& +\left(1-\alpha_{n}\right) \beta_{n}\|S z-z\|\left\|x_{n+1}-z\right\| \\
& \leq \frac{1-\alpha_{n}(1-\rho)}{2}\left(\left\|x_{n}-z\right\|^{2}+\left\|x_{n+1}-z\right\|^{2}\right) \\
& +\alpha_{n}\left\langle f(z)-z, x_{n+1}-z\right\rangle \\
& +\left(1-\alpha_{n}\right) \beta_{n}\|S z-z\|\left\|x_{n+1}-z\right\|
\end{aligned}
$$

which implies that

$$
\begin{aligned}
\left\|x_{n+1}-z\right\|^{2} \leq( & \left.1-\frac{2 \alpha_{n}(1-\rho)}{1+\alpha_{n}(1-\rho)}\right)\left\|x_{n}-z\right\|^{2} \\
& +\frac{2 \alpha_{n}}{1+\alpha_{n}(1-\rho)}\left\langle f(z)-z, x_{n+1}-z\right\rangle
\end{aligned}
$$

$$
\begin{gathered}
+\frac{2\left(1-\alpha_{n}\right) \beta_{n}}{1+\alpha_{n}(1-\rho)}\|S z-z\|\left\|x_{n+1}-z\right\| \\
\leq\left(1-\frac{2 \alpha_{n}(1-\rho)}{1+\alpha_{n}(1-\rho)}\right)\left\|x_{n}-z\right\|^{2} \\
+\frac{2 \alpha_{n}(1-\rho)}{1+\alpha_{n}(1-\rho)}\left\{\frac{1}{1-\rho}\left\langle f(z)-z, x_{n+1}-z\right\rangle\right. \\
+\frac{\left(1-\alpha_{n}\right) \beta_{n}}{\alpha_{n}(1-\rho)}\|S z-z\| \\
\left.\times\left\|x_{n+1}-z\right\|\right\} .
\end{gathered}
$$

Let $\gamma_{n}=2 \alpha_{n}(1-\rho) /\left(1+\alpha_{n}(1-\rho)\right)$ and $\delta_{n}=\left(2 \alpha_{n}(1-\rho) /(1+\right.$ $\left.\left.\alpha_{n}(1-\rho)\right)\right)\left\{(1 /(1-\rho))\left\langle f(z)-z, x_{n+1}-z\right\rangle+\left(\left(1-\alpha_{n}\right) \beta_{n} /\left(\alpha_{n}(1-\right.\right.\right.$ $\left.\rho)))\|S z-z\|\left\|x_{n+1}-z\right\|\right\}$.

Since

$$
\begin{aligned}
\sum_{n=1}^{\infty} \alpha_{n}=\infty, \quad 1+\alpha_{n}(1-\rho) \leq 2, \\
\limsup _{n \rightarrow \infty}\left\{\frac{1}{1-\rho}\left\langle f(z)-z, x_{n+1}-z\right\rangle\right. \\
\left.+\frac{\left(1-\alpha_{n}\right) \beta_{n}}{\alpha_{n}(1-\rho)}\|S z-z\|\left\|x_{n+1}-z\right\|\right\} \leq 0 .
\end{aligned}
$$

It follows that

$$
\sum_{n=1}^{\infty} \gamma_{n}=\infty \quad \limsup _{n \rightarrow \infty} \frac{\delta_{n}}{\gamma_{n}} \lim \leq 0
$$

Thus, all the conditions of Lemma 7 are satisfied. Hence, we deduce that $x_{n} \rightarrow z$.

Since $P_{S^{*} \cap M E P(F) \cap F(T)} f$ is a contraction, there exists a unique $z \in C$ such that $z=P_{S^{*} \cap \operatorname{MEP}(F) \cap F(T)} f(z)$. From (20), it follows that $z$ is the unique solution of the problem (74). This completes the proof.

Theorem 15. Let $C$ be a nonempty closed convex subset of a real Hilbert space $H$. Let $D, B_{i}: C \rightarrow H$ be $\eta, \theta_{i}$-inverse strongly monotone mappings for each $i=1,2$, respectively. Let $F: C \times C \rightarrow \mathbb{R}$ be a bifunction satisfying the assumptions (i)-(iv) of Lemma 4, S:C $\rightarrow \mathrm{H}$ a nonexpansive mapping, and $\left\{T_{i}\right\}_{i=1}^{\infty}: C \rightarrow C$ a countable family of $k_{i}$-strict pseudocontraction mappings such that $S^{*} \cap \operatorname{MEP}(F) \cap F(T) \neq \emptyset$, where $F(T):=\bigcap_{i=1}^{\infty} F\left(T_{i}\right)=\bigcap_{i=1}^{\infty} F\left(V_{i}\right)$. Let $f$ be a $\rho$ contraction mapping. For a given $x_{0} \in C$ arbitrarily, let the iterative sequences $\left\{u_{n}\right\},\left\{x_{n}\right\},\left\{y_{n}\right\}$, and $\left\{z_{n}\right\}$ be generated by

$$
\begin{array}{r}
F\left(u_{n}, y\right)+\left\langle D x_{n}, y-u_{n}\right\rangle+\frac{1}{r_{n}}\left\langle y-u_{n}, u_{n}-x_{n}\right\rangle \geq 0, \\
\forall y \in C ; \\
z_{n}=P_{C}\left[P_{C}\left[u_{n}-\mu_{2} B_{2} u_{n}\right]-\mu_{1} B_{1} P_{C}\left[u_{n}-\mu_{2} B_{2} u_{n}\right]\right] ;
\end{array}
$$




$$
\begin{gathered}
y_{n}=\beta_{n} S x_{n}+\left(1-\beta_{n}\right) z_{n} ; \\
x_{n+1}=P_{C}\left[\alpha_{n} f\left(x_{n}\right)+\sum_{i=1}^{n}\left(\alpha_{i-1}-\alpha_{i}\right) V_{i} y_{n}\right], \quad \forall n \geq 0,
\end{gathered}
$$

where $V_{i}=k_{i} I+\left(1-k_{i}\right) T_{i}, 0 \leq k_{i}<1, \mu_{i} \in\left(0,2 \theta_{i}\right)$ for each $i=1,2,\left\{r_{n}\right\} \subset(0,2 \eta), \alpha_{0}=1,\left\{\alpha_{n}\right\}$ is a strictly decreasing sequence in $(0,1)$, and $\left\{\beta_{n}\right\}$ is a sequence in $(0,1)$ satisfying the following conditions:

(a) $\lim _{n \rightarrow \infty} \alpha_{n}=0$ and $\sum_{n=1}^{\infty} \alpha_{n}=\infty$,

(b) $\lim _{n \rightarrow \infty}\left(\beta_{n} / \alpha_{n}\right)=\tau \in(0, \infty)$,

(c) $\sum_{n=1}^{\infty}\left(\alpha_{n-1}-\alpha_{n}\right)<\infty$ and $\sum_{n=1}^{\infty}\left|\beta_{n-1}-\beta_{n}\right|<\infty$,

(d) $\lim _{n \rightarrow \infty}\left((1 / \mu)\left|r_{n-1}-r_{n}\right|+\left|\alpha_{n-1}-\alpha_{n}\right|+\mid \beta_{n-1}-\right.$ $\left.\beta_{n} \mid / \alpha_{n} \beta_{n}\right)=0$,

(e) there exists a constant $K>0$ such that $\left(1 / \alpha_{n}\right) \mid\left(1 / \beta_{n}\right)-$ $\left(1 / \beta_{n-1}\right) \mid \leq K$,

(f) $\liminf \operatorname{in}_{n \rightarrow \infty} r_{n}<\lim \sup _{n \rightarrow \infty} r_{n}<2 \eta$ and $\sum_{n=1}^{\infty} \mid r_{n-1}-$ $r_{n} \mid<\infty$.

Then, sequence $\left\{x_{n}\right\}$ generated by Algorithm (89) converges strongly to $x^{*} \in S^{*} \cap \operatorname{MEP}(F) \cap F(T)$, which is the unique solution of the variational inequality

$$
\left\langle\frac{1}{\tau}(I-f) x^{*}+(I-S) x^{*}, x-x^{*}\right\rangle \geq 0,
$$

$\forall x \in S^{*} \cap \operatorname{MEP}(F) \cap F(T)$.

Proof. From $\lim _{n \rightarrow \infty}\left(\beta_{n} / \alpha_{n}\right)=\tau \in(0, \infty)$, without loss of generality, we can assume that $\beta_{n} \leq(1+\tau) \alpha_{n}$ for all $n \geq 1$. Hence $\beta_{n} \rightarrow 0$. By similar argument as that lemmas 12 and 13 , we can deduce that $\left\{x_{n}\right\}$ is bounded, $\lim _{n \rightarrow \infty}\left\|x_{n+1}-x_{n}\right\|=$ $0, \lim _{n \rightarrow \infty}\left\|x_{n}-z_{n}\right\|=0$ (see (67)), and $\left\|\left(I-V_{i}\right) x_{n}\right\| \rightarrow 0$. Then, we have

$$
\begin{aligned}
\left\|y_{n}-x_{n}\right\| \leq & \beta_{n}\left\|x_{n}-S x_{n}\right\| \\
& +\left(1-\beta_{n}\right)\left\|x_{n}-z_{n}\right\| \longrightarrow 0 \quad \text { as } n \longrightarrow \infty .
\end{aligned}
$$

It follows that, for all $i \geq 1$,

$$
\begin{array}{r}
\left\|y_{n}-V_{i} x_{n}\right\| \leq\left\|y_{n}-x_{n}\right\|+\left\|x_{n}-V_{i} x_{n}\right\| \\
\text { as } n \longrightarrow 0
\end{array}
$$

From (91) and (92), we have

$$
\begin{aligned}
\left\|y_{n}-V_{i} y_{n}\right\| & \leq\left\|y_{n}-V_{i} x_{n}\right\|+\left\|V_{i} x_{n}-V_{i} y_{n}\right\| \\
& \leq\left\|y_{n}-V_{i} x_{n}\right\|+\left\|y_{n}-x_{n}\right\| \longrightarrow 0 \quad \text { as } n \longrightarrow \infty
\end{aligned}
$$

Set $w_{n}=\alpha_{n} f\left(x_{n}\right)+\sum_{i=1}^{n}\left(\alpha_{i-1}-\alpha_{i}\right) V_{i} y_{n}$. From (47) and (48), we obtain

$$
\begin{aligned}
& \frac{\left\|x_{n+1}-x_{n}\right\|}{\beta_{n}} \leq \frac{\left\|w_{n}-w_{n-1}\right\|}{\beta_{n}} \\
& \leq\left(1-(1-\rho) \alpha_{n}\right) \frac{\left\|x_{n}-x_{n-1}\right\|}{\beta_{n}} \\
& +M\left(\frac{(1 / \mu)\left|r_{n}-r_{n-1}\right|}{\beta_{n}}+\frac{\left|\beta_{n}-\beta_{n-1}\right|}{\beta_{n}}\right. \\
& \left.+\frac{\left|\alpha_{n}-\alpha_{n-1}\right|}{\beta_{n}}\right) \\
& =\left(1-(1-\rho) \alpha_{n}\right) \frac{\left\|x_{n}-x_{n-1}\right\|}{\beta_{n-1}} \\
& +\left(1-(1-\rho) \alpha_{n}\right)\left\|x_{n}-x_{n-1}\right\|\left(\frac{1}{\beta_{n}}-\frac{1}{\beta_{n-1}}\right) \\
& +M\left(\frac{(1 / \mu)\left|r_{n}-r_{n-1}\right|}{\beta_{n}}+\frac{\left|\beta_{n}-\beta_{n-1}\right|}{\beta_{n}}\right. \\
& \left.+\frac{\left|\alpha_{n}-\alpha_{n-1}\right|}{\beta_{n}}\right) \\
& \leq\left(1-(1-\rho) \alpha_{n}\right) \frac{\left\|x_{n}-x_{n-1}\right\|}{\beta_{n-1}} \\
& +\left\|x_{n}-x_{n-1}\right\|\left|\frac{1}{\beta_{n}}-\frac{1}{\beta_{n-1}}\right| \\
& +M\left(\frac{(1 / \mu)\left|r_{n}-r_{n-1}\right|}{\beta_{n}}+\frac{\left|\beta_{n}-\beta_{n-1}\right|}{\beta_{n}}\right. \\
& \left.+\frac{\left|\alpha_{n}-\alpha_{n-1}\right|}{\beta_{n}}\right) \\
& \leq\left(1-(1-\rho) \alpha_{n}\right) \frac{\left\|x_{n}-x_{n-1}\right\|}{\beta_{n-1}} \\
& +\alpha_{n} K\left\|x_{n}-x_{n-1}\right\| \\
& +M\left(\frac{(1 / \mu)\left|r_{n}-r_{n-1}\right|}{\beta_{n}}+\frac{\left|\beta_{n}-\beta_{n-1}\right|}{\beta_{n}}\right. \\
& \left.+\frac{\left|\alpha_{n}-\alpha_{n-1}\right|}{\beta_{n}}\right) \\
& \leq\left(1-(1-\rho) \alpha_{n}\right) \frac{\left\|w_{n-1}-w_{n-2}\right\|}{\beta_{n-1}} \\
& +\alpha_{n} K\left\|x_{n}-x_{n-1}\right\| \\
& +M\left(\frac{(1 / \mu)\left|r_{n}-r_{n-1}\right|}{\beta_{n}}+\frac{\left|\beta_{n}-\beta_{n-1}\right|}{\beta_{n}}\right. \\
& \left.+\frac{\left|\alpha_{n}-\alpha_{n-1}\right|}{\beta_{n}}\right) .
\end{aligned}
$$


Let $\gamma_{n}=(1-\rho) \alpha_{n}$ and $\delta_{n}=\alpha_{n} K\left\|x_{n}-x_{n-1}\right\|+M\left((1 / \mu) \mid r_{n}-\right.$ $\left.r_{n-1}\left|/ \beta_{n}+\right| \beta_{n}-\beta_{n-1}\left|/ \beta_{n}+\right| \alpha_{n}-\alpha_{n-1} \mid / \beta_{n}\right)$. From conditions (a) and (d), we have

$$
\sum_{n=1}^{\infty} \gamma_{n}=\infty, \quad \lim _{n \rightarrow \infty} \frac{\delta_{n}}{\gamma_{n}}=0
$$

By Lemma 7, we obtain

$$
\begin{gathered}
\lim _{n \rightarrow \infty} \frac{\left\|x_{n+1}-x_{n}\right\|}{\beta_{n}}=0, \\
\lim _{n \rightarrow \infty} \frac{\left\|w_{n+1}-w_{n}\right\|}{\beta_{n}}=\lim _{n \rightarrow \infty} \frac{\left\|w_{n+1}-w_{n}\right\|}{\alpha_{n}}=0 .
\end{gathered}
$$

From (89), we have

$$
\begin{aligned}
x_{n+1}= & P_{C}\left[w_{n}\right]-w_{n}+\alpha_{n} f\left(x_{n}\right) \\
& +\sum_{i=1}^{n}\left(\alpha_{i-1}-\alpha_{i}\right)\left(V_{i} y_{n}-y_{n}\right)+\left(1-\alpha_{n}\right) y_{n} .
\end{aligned}
$$

Hence, it follows that

$$
\begin{aligned}
x_{n}-x_{n+1}= & \left(1-\alpha_{n}\right) x_{n}+\alpha_{n} x_{n} \\
& -\left(P_{C}\left[w_{n}\right]-w_{n}+\alpha_{n} f\left(x_{n}\right)\right. \\
& \left.\quad+\sum_{i=1}^{n}\left(\alpha_{i-1}-\alpha_{i}\right)\left(V_{i} y_{n}-y_{n}\right)+\left(1-\alpha_{n}\right) y_{n}\right) \\
= & \left(1-\alpha_{n}\right)\left[\beta_{n}\left(x_{n}-S x_{n}\right)+\left(1-\beta_{n}\right)\left(x_{n}-z_{n}\right)\right] \\
& +\left(w_{n}-P_{C}\left[w_{n}\right]\right) \\
& +\sum_{i=1}^{n}\left(\alpha_{i-1}-\alpha_{i}\right)\left(y_{n}-V_{i} y_{n}\right)+\alpha_{n}\left(x_{n}-f\left(x_{n}\right)\right)
\end{aligned}
$$

and hence

$$
\begin{aligned}
\frac{x_{n}-x_{n+1}}{\left(1-\alpha_{n}\right) \beta_{n}}= & x_{n}-S x_{n}+\frac{\left(1-\beta_{n}\right)}{\beta_{n}}\left(x_{n}-z_{n}\right) \\
& +\frac{1}{\left(1-\alpha_{n}\right) \beta_{n}}\left(w_{n}-P_{C}\left[w_{n}\right]\right) \\
& +\frac{1}{\left(1-\alpha_{n}\right) \beta_{n}} \sum_{i=1}^{n}\left(\alpha_{i-1}-\alpha_{i}\right)\left(y_{n}-V_{i} y_{n}\right) \\
& +\frac{\alpha_{n}}{\left(1-\alpha_{n}\right) \beta_{n}}\left(x_{n}-f\left(x_{n}\right)\right) .
\end{aligned}
$$

Let $v_{n}=\left(x_{n}-x_{n+1}\right) /\left(1-\alpha_{n}\right) \beta_{n}$. For any $z \in S^{*} \cap \operatorname{MEP}(F) \cap$ $F(T)$, we have

$$
\begin{aligned}
\left\langle v_{n}, x_{n}-z\right\rangle & \\
= & \frac{1}{\left(1-\alpha_{n}\right) \beta_{n}}\left\langle w_{n}-P_{C}\left[w_{n}\right], P_{C}\left[w_{n-1}\right]-z\right\rangle \\
& +\frac{\alpha_{n}}{\left(1-\alpha_{n}\right) \beta_{n}}\left\langle(I-f) x_{n}, x_{n}-z\right\rangle \\
& +\left\langle x_{n}-S x_{n}, x_{n}-z\right\rangle \\
& +\frac{\left(1-\beta_{n}\right)}{\beta_{n}}\left\langle x_{n}-z_{n}, x_{n}-z\right\rangle \\
& +\frac{1}{\left(1-\alpha_{n}\right) \beta_{n}} \sum_{i=1}^{n}\left(\alpha_{i-1}-\alpha_{i}\right)\left\langle y_{n}-V_{i} y_{n}, x_{n}-z\right\rangle .
\end{aligned}
$$

Since $S$ is nonexpansive mapping, $f$ is $\rho$-contraction mapping and $V_{i}$ is $k_{i}$-strict pseudocontraction mapping. Then, $(I-S)$ and $\left(I-V_{i}\right)$ are monotones, and $f$ is strongly monotone with coefficient $(1-\rho)$. We can deduce that

$$
\begin{aligned}
\left\langle x_{n}-\right. & \left.S x_{n}, x_{n}-z\right\rangle \\
= & \left\langle(I-S) x_{n}-(I-S) z, x_{n}-z\right\rangle \\
& +\left\langle(I-S) z, x_{n}-z\right\rangle \\
\geq & \left\langle(I-S) z, x_{n}-z\right\rangle, \\
\langle(I- & \left.f) x_{n}, x_{n}-z\right\rangle \\
= & \left\langle(I-f) x_{n}-(I-f) z, x_{n}-z\right\rangle \\
& +\left\langle(I-f) z, x_{n}-z\right\rangle \geq(1-\rho)\left\|x_{n}-z\right\|^{2} \\
& +\left\langle(I-f) z, x_{n}-z\right\rangle, \\
\langle(I- & \left.\left.V_{i}\right) y_{n}, x_{n}-z\right\rangle \\
= & \left\langle\left(I-V_{i}\right) y_{n}-\left(I-V_{i}\right) z, x_{n}-y_{n}\right\rangle \\
& +\left\langle\left(I-V_{i}\right) y_{n}-\left(I-V_{i}\right) z, y_{n}-z\right\rangle \\
\geq & \left\langle\left(I-V_{i}\right) y_{n}-\left(I-V_{i}\right) z, x_{n}-y_{n}\right\rangle \\
= & \left\langle\left(I-V_{i}\right) y_{n}, x_{n}-y_{n}\right\rangle \\
= & \left\langle\left(I-V_{i}\right) y_{n}, \beta_{n}\left(x_{n}-S x_{n}\right)+\left(1-\beta_{n}\right)\left(x_{n}-z_{n}\right)\right\rangle .
\end{aligned}
$$

From (20), we get

$$
\begin{aligned}
\left\langle w_{n}\right. & \left.-P_{C}\left[w_{n}\right], P_{C}\left[w_{n-1}\right]-z\right\rangle \\
= & \left\langle w_{n}-P_{C}\left[w_{n}\right], P_{C}\left[w_{n-1}\right]-P_{C}\left[w_{n}\right]\right\rangle \\
& +\left\langle w_{n}-P_{C}\left[w_{n}\right], P_{C}\left[w_{n}\right]-z\right\rangle \\
\geq & \left\langle w_{n}-P_{C}\left[w_{n}\right], P_{C}\left[w_{n-1}\right]-P_{C}\left[w_{n}\right]\right\rangle .
\end{aligned}
$$


Then, from (100)-(102), we have

$$
\begin{aligned}
\left\langle v_{n}, x_{n}-z\right\rangle \geq & \frac{1}{\left(1-\alpha_{n}\right) \beta_{n}} \\
& \times\left\langle w_{n}-P_{C}\left[w_{n}\right], P_{C}\left[w_{n-1}\right]-P_{C}\left[w_{n}\right]\right\rangle \\
& +\frac{\alpha_{n}}{\left(1-\alpha_{n}\right) \beta_{n}}\left\langle(I-f) z, x_{n}-z\right\rangle \\
& +\left\langle(I-S) z, x_{n}-z\right\rangle \\
& +\frac{\left(1-\beta_{n}\right)}{\beta_{n}}\left\langle x_{n}-z_{n}, x_{n}-z\right\rangle \\
& +\frac{\left(1-\beta_{n}\right)}{\left(1-\alpha_{n}\right) \beta_{n}} \sum_{i=1}^{n}\left(\alpha_{i-1}-\alpha_{i}\right) \\
& \times\left\langle\left(I-V_{i}\right) y_{n}, x_{n}-z_{n}\right\rangle \\
& +\frac{1}{\left(1-\alpha_{n}\right)} \sum_{i=1}^{n}\left(\alpha_{i-1}-\alpha_{i}\right) \\
& +\frac{(1-\rho) \alpha_{n}}{\left(1-\alpha_{n}\right) \beta_{n}}\left\|x_{n}-z\right\|^{2} . \\
& \times\left\langle\left(I-V_{i}\right) y_{n}, x_{n}-S x_{n}\right\rangle
\end{aligned}
$$

Then, we obtain

$$
\begin{aligned}
\left\|x_{n}-z\right\|^{2} \leq & \frac{1}{(1-\rho) \alpha_{n}}\left\|w_{n}-P_{C}\left[w_{n}\right]\right\|\left\|w_{n-1}-w_{n}\right\| \\
& -\frac{1}{(1-\rho)}\left\langle(I-f) z, x_{n}-z\right\rangle \\
& +\frac{\left(1-\alpha_{n}\right) \beta_{n}}{(1-\rho) \alpha_{n}}\left(\left\langle v_{n}, x_{n}-z\right\rangle\right. \\
& -\frac{\left(1-\beta_{n}\right)\left(1-\alpha_{n}\right)}{(1-\rho) \alpha_{n}}\left\langle x_{n}-z_{n}, x_{n}-z\right\rangle \\
& -\frac{\left(1-\beta_{n}\right)}{(1-\rho) \alpha_{n}} \sum_{i=1}^{n}\left(\alpha_{i-1}-\alpha_{i}\right)\left\langle\left(I-V_{i}\right) y_{n}, x_{n}-z_{n}\right\rangle \\
& -\frac{\beta_{n}}{(1-\rho) \alpha_{n}} \sum_{i=1}^{n}\left(\alpha_{i-1}-\alpha_{i}\right) \\
& +\frac{\left(1-\alpha_{n}\right) \beta_{n}}{(1-\rho) \alpha_{n}} \\
\leq & \frac{1 w_{n-1}-w_{n} \|}{(1-\rho) \alpha_{n}}\left\|w_{n}-P_{C}\left[w_{n}\right]\right\| \\
& -\left\langle\left(I-V_{i}\right) y_{n}, x_{n}-S x_{n}\right\rangle \\
& \\
& \\
& \\
& \\
& \\
&
\end{aligned}
$$

$$
\begin{aligned}
& \times\left(\left\langle v_{n}, x_{n}-z\right\rangle-\left\langle(I-S) z, x_{n}-z\right\rangle\right) \\
& +\frac{1}{(1-\rho)} \frac{\left(1-\beta_{n}\right)}{\beta_{n}} \frac{\beta_{n}}{\alpha_{n}}\left\|x_{n}-z_{n}\right\|\left\|x_{n}-z\right\| \\
& +\frac{1}{(1-\rho)} \frac{\left(1-\beta_{n}\right)}{\beta_{n}} \frac{\beta_{n}}{\alpha_{n}} \\
& \times \sum_{i=1}^{n}\left(\alpha_{i-1}-\alpha_{i}\right)\left\|\left(I-V_{i}\right) y_{n}\right\|\left\|x_{n}-z_{n}\right\| \\
& -\frac{\beta_{n}}{(1-\rho) \alpha_{n}} \\
& \times \sum_{i=1}^{n}\left(\alpha_{i-1}-\alpha_{i}\right)\left\langle\left(I-V_{i}\right) y_{n}, x_{n}-S x_{n}\right\rangle .
\end{aligned}
$$

By condition (e) of Theorem 15, there exists a constant $N>$ 0 such that $\left(\left(1-\beta_{n}\right) / \beta_{n}\right) \leq N$. Since $\lim _{n \rightarrow \infty}\left\|x_{n}-z_{n}\right\|=$ $0, v_{n} \rightarrow 0,\left(I-V_{i}\right) y_{n} \rightarrow 0$, and $\left\|w_{n-1}-w_{n}\right\| / \alpha_{n} \rightarrow 0$ as $n \rightarrow \infty$, then every weak cluster point of $\left\{x_{n}\right\}$ is also a strong cluster point. Since $\left\{x_{n}\right\}$ is bounded, by Lemma 13 there exists a subsequence $\left\{x_{n_{k}}\right\}$ of $\left\{x_{n}\right\}$ converging to a point $x^{*} \in F(T)$; in similar argument as that Theorem 14 we can show that $x^{*} \in$ $S^{*} \cap \operatorname{MEP}(F) \cap F(T)$.

From (100)-(102), it follows that, for any $z \in S^{*} \cap$ $\operatorname{MEP}(F) \cap F(T)$,

$$
\begin{aligned}
\langle(I- & \left.f) x_{n_{k}}, x_{n_{k}}-z\right\rangle \\
= & \frac{\left(1-\alpha_{n_{k}}\right) \beta_{n_{k}}}{\alpha_{n_{k}}}\left\langle v_{n_{k}}, x_{n_{k}}-z\right\rangle-\frac{1}{\alpha_{n_{k}}} \\
& \times\left\langle w_{n_{k}}-P_{C}\left[w_{n_{k}}\right], P_{C}\left[w_{n_{k}-1}\right]-z\right\rangle \\
& -\frac{\left(1-\alpha_{n_{k}}\right) \beta_{n_{k}}}{\alpha_{n_{k}}}\left\langle x_{n_{k}}-S x_{n_{k}}, x_{n_{k}}-z\right\rangle \\
& -\frac{\left(1-\alpha_{n_{k}}\right)\left(1-\beta_{n_{k}}\right)}{\alpha_{n_{k}}}\left\langle x_{n_{k}}-z_{n_{k}}, x_{n_{k}}-z\right\rangle \\
& -\frac{1}{\alpha_{n_{k}}} \sum_{i=1}^{n}\left(\alpha_{i-1}-\alpha_{i}\right)\left\langle y_{n_{k}}-V_{i} y_{n_{k}}, x_{n_{k}}-z\right\rangle \\
\leq & \frac{\left(1-\alpha_{n_{k}}\right) \beta_{n_{k}}}{\alpha_{n_{k}}}\left\langle v_{n_{k}}, x_{n_{k}}-z\right\rangle \\
& \times \frac{1}{\alpha_{n_{k}}}\left\|w_{n_{k}}-P_{C}\left[w_{n_{k}}\right]\right\| \\
& \times\left\|w_{n_{k}-1}-S w_{n_{k}}\right\|-\frac{\left(1-\alpha_{n_{k}}\right) \beta_{n_{k}}}{\alpha_{n_{k}}} \\
& \left.x_{n_{k}}-z\right\rangle \\
& \\
& \\
& \\
& \\
& \\
&
\end{aligned}
$$




$$
\begin{aligned}
& +\frac{\left(1-\beta_{n_{k}}\right)}{\beta_{n_{k}}} \frac{\beta_{n_{k}}}{\alpha_{n_{k}}}\left\|x_{n_{k}}-z_{n_{k}}\right\| \\
& \times\left\|x_{n_{k}}-z\right\|+\frac{\left(1-\beta_{n_{k}}\right)}{\beta_{n_{k}}} \frac{\beta_{n_{k}}}{\alpha_{n_{k}}} \\
& \times \sum_{i=1}^{n_{k}}\left(\alpha_{i-1}-\alpha_{i}\right)\left\|\left(I-V_{i}\right) y_{n_{k}}\right\|\left\|x_{n_{k}}-z_{n_{k}}\right\| \\
& -\frac{\beta_{n_{k}}}{\alpha_{n_{k}}} \sum_{i=1}^{n_{k}}\left(\alpha_{i-1}-\alpha_{i}\right)\left\langle\left(I-V_{i}\right) y_{n_{k}}, x_{n_{k}}-S x_{n_{k}}\right\rangle .
\end{aligned}
$$

Since $\lim _{n \rightarrow \infty}\left\|x_{n}-z_{n}\right\|=0, v_{n} \rightarrow 0,\left(I-V_{i}\right) y_{n} \rightarrow 0$, and $\left\|w_{n-1}-w_{n}\right\| / \alpha_{n} \rightarrow 0$; letting $k \rightarrow \infty$ in (105), we obtain

$$
\left\langle(I-f) x^{*}, x^{*}-z\right\rangle \leq-\tau\left\langle x^{*}-S x^{*}, x^{*}-z\right\rangle ;
$$

that is,

$$
\left\langle\frac{1}{\tau}(I-f) x^{*}+(I-S) x^{*}, z-x^{*}\right\rangle \geq 0 .
$$

In the following, we show that (90) has unique solution. Assume that $x^{\prime}$ is another solution. Then, we have

$$
\begin{aligned}
& \left\langle(I-f) x^{\prime}, x^{\prime}-x^{*}\right\rangle \leq-\tau\left\langle x^{\prime}-S x^{\prime}, x^{\prime}-x^{*}\right\rangle, \\
& \left\langle(I-f) x^{*}, x^{*}-x^{\prime}\right\rangle \leq-\tau\left\langle x^{*}-S x^{*}, x^{*}-x^{\prime}\right\rangle .
\end{aligned}
$$

Adding (108), we get

$$
\begin{aligned}
(1-\rho)\left\|x^{\prime}-x^{*}\right\|^{2} & \leq\left\langle(I-f) x^{\prime}-(I-f) x^{*}, x^{\prime}-x^{*}\right\rangle \\
& \leq-\tau\left\langle(I-S) x^{\prime}-(I-S) x^{*}, x^{\prime}-x^{*}\right\rangle \leq 0 .
\end{aligned}
$$

Then, $x^{\prime}=x^{*}$. Since (90) has unique solution, it follows that $w_{w}\left(x_{n}\right)=\left\{x^{*}\right\}$. Since every weak cluster point of $\left\{x_{n}\right\}$ is also a strong cluster point, we conclude that $\left\{x_{n}\right\} \rightarrow x^{*}$. This completes the proof.

\section{Applications}

In this section, we obtain the following results by using a special case of the proposed method. The first result can be viewed as extension and improvement of the method of $\mathrm{Gu}$ et al. [11] for finding the approximate element of the common set of solutions of a generalized equilibrium problem and a hierarchical fixed point problem in a real Hilbert space.

Corollary 16. Let $C$ be a nonempty closed convex subset of a real Hilbert space $H$. Let $D: C \rightarrow H$ be $\eta$-inverse strongly monotone mappings, respectively. Let $F: C \times C \rightarrow \mathbb{R}$ be a bifunction satisfying the assumptions (i)-(iv) of Lemma 4, S : $C \rightarrow H$ a nonexpansive mapping, and $\left\{T_{i}\right\}_{i=1}^{\infty}: C \rightarrow C a$ countable family of $k_{i}$-strict pseudocontraction mappings such that $F(T) \cap \operatorname{MEP}(F) \neq \emptyset$, where $F(T)=\cap_{i=1}^{\infty} F\left(T_{i}\right)$. Let $f$ be a $\rho$-contraction mapping. For a given $x_{0} \in C$ arbitrarily, let the iterative sequences $\left\{u_{n}\right\},\left\{x_{n}\right\},\left\{y_{n}\right\}$, and $\left\{z_{n}\right\}$ be generated by

$$
\begin{array}{cc}
F\left(u_{n}, y\right)+\left\langle D x_{n}, y-u_{n}\right\rangle+\frac{1}{r_{n}}\left\langle y-u_{n}, u_{n}-x_{n}\right\rangle \geq 0, & \forall y \in C ; \\
y_{n}=\beta_{n} S x_{n}+\left(1-\beta_{n}\right) u_{n} ; & \\
x_{n+1}=P_{C}\left[\alpha_{n} f\left(x_{n}\right)+\sum_{i=1}^{n}\left(\alpha_{i-1}-\alpha_{i}\right) T_{i} y_{n}\right], & \forall n \geq 0,
\end{array}
$$

where $\alpha_{0}=1,\left\{\alpha_{n}\right\}$ is a strictly decreasing sequence in $(0,1)$, and $\left\{\beta_{n}\right\}$ is a sequence in $(0,1)$ satisfying the following conditions:

(a) $\lim _{n \rightarrow \infty} \alpha_{n}=0$ and $\sum_{n=1}^{\infty} \alpha_{n}=\infty$,

(b) $\lim _{n \rightarrow \infty}\left(\beta_{n} / \alpha_{n}\right)=\tau \in(0, \infty)$,

(c) $\sum_{n=1}^{\infty}\left(\alpha_{n-1}-\alpha_{n}\right)<\infty$ and $\sum_{n=1}^{\infty}\left|\beta_{n-1}-\beta_{n}\right|<\infty$,

(d) $\lim _{n \rightarrow \infty}\left((1 / \mu)\left(\left|r_{n}-r_{n-1}\right|+\left|\alpha_{n-1}-\alpha_{n}\right|+\mid \beta_{n-1}-\right.\right.$ $\left.\left.\beta_{n} \mid\right) /\left(\alpha_{n} \beta_{n}\right)\right)=0$,

(e) there exists a constant $K>0$ such that $\left(1 / \alpha_{n}\right) \mid\left(1 / \beta_{n}\right)-$ $\left(1 / \beta_{n-1}\right) \mid \leq K$,

(f) $\liminf _{n \rightarrow \infty} r_{n}>0$ and $\sum_{n=1}^{\infty}\left|r_{n-1}-r_{n}\right|<\infty$.

Then, sequence $\left\{x_{n}\right\}$ generated by Algorithm (110) converges strongly to $x^{*} \in \operatorname{MEP}(F) \cap F(T)$, which is the unique solution of the variational inequality

$$
\left\langle\frac{1}{\tau}(I-f) x^{*}+(I-S) x^{*}, x-x^{*}\right\rangle \geq 0
$$

$$
\forall x \in \operatorname{MEP}(F) \cap F(T) .
$$

Proof. Putting $B_{1}=B_{2}=0$ and $k_{i}=0$, for all $i \geq 1$ in Theorem 15, then conclusion of Corollary 16 is obtained.

The following result can be viewed as extension and improvement of the method of Yao et al. [31] for finding the approximate element of the common set of solutions of a generalized equilibrium problem and a hierarchical fixed point problem in a real Hilbert space.

Corollary 17. Let $C$ be a nonempty closed convex subset of a real Hilbert space $H$. Let $D: C \rightarrow H$ be $\eta$-inverse strongly monotone mappings, respectively. Let $F: C \times C \rightarrow \mathbb{R}$ be a bifunction satisfying the assumptions (i)-(iv) of Lemma 4, $S: C \rightarrow H$ a nonexpansive mapping, and $T: C \rightarrow C$ a countable family of $k$-strict pseudocontraction mappings such that $F(T) \cap \operatorname{MEP}(F) \neq \emptyset$. Let $f$ be a $\rho$-contraction mapping. For a given $x_{0} \in C$ arbitrarily, let the iterative sequences $\left\{u_{n}\right\},\left\{x_{n}\right\},\left\{y_{n}\right\}$, and $\left\{z_{n}\right\}$ be generated by

$$
\begin{array}{cc}
F\left(u_{n}, y\right)+\left\langle D x_{n}, y-u_{n}\right\rangle+\frac{1}{r_{n}}\left\langle y-u_{n}, u_{n}-x_{n}\right\rangle \geq 0, & \forall y \in C ; \\
y_{n}=\beta_{n} S x_{n}+\left(1-\beta_{n}\right) u_{n} ; & \\
x_{n+1}=P_{C}\left[\alpha_{n} f\left(x_{n}\right)+\left(1-\alpha_{n}\right) T y_{n}\right], & \forall n \geq 0,
\end{array}
$$


where $\alpha_{0}=1,\left\{\alpha_{n}\right\}$ is a strictly decreasing sequence in $(0,1)$ and $\left\{\beta_{n}\right\}$ is a sequence in $(0,1)$ satisfying the following conditions:

(a) $\lim _{n \rightarrow \infty} \alpha_{n}=0$ and $\sum_{n=1}^{\infty} \alpha_{n}=\infty$,

(b) $\lim _{n \rightarrow \infty}\left(\beta_{n} / \alpha_{n}\right)=\tau \in(0, \infty)$,

(c) $\sum_{n=1}^{\infty}\left(\alpha_{n-1}-\alpha_{n}\right)<\infty$ and $\sum_{n=1}^{\infty}\left|\beta_{n-1}-\beta_{n}\right|<\infty$,

(d) $\lim _{n \rightarrow \infty}\left((1 / \mu)\left|r_{n}-r_{n-1}\right|+\left|\alpha_{n-1}-\alpha_{n}\right|+\mid \beta_{n-1}-\right.$ $\left.\beta_{n} \mid\right) / \alpha_{n} \beta_{n}=0$,

(e) there exists a constant $K>0$ such that $\left(1 / \alpha_{n}\right) \mid\left(1 / \beta_{n}\right)-$ $\left(1 / \beta_{n-1}\right) \mid \leq K$,

(f) $\liminf _{n \rightarrow \infty} r_{n}>0$ and $\sum_{n=1}^{\infty}\left|r_{n-1}-r_{n}\right|<\infty$.

Then, sequence $\left\{x_{n}\right\}$ generated by Algorithm (112) converges strongly to $x^{*} \in \operatorname{MEP}(F) \cap F(T)$, which is the unique solution of the variational inequality

$$
\begin{array}{r}
\left\langle\frac{1}{\tau}(I-f) x^{*}+(I-S) x^{*}, x-x^{*}\right\rangle \geq 0, \\
\forall x \in \operatorname{MEP}(F) \cap F(T) .
\end{array}
$$

Proof. Putting $B_{1}=B_{2}=0, k_{i}=0$, and $T_{i}=T$ for all $i \geq 1$ in Theorem 15, then conclusion of Corollary 17 is obtained.

\section{Conclusions}

In this paper, we suggest and analyze an iterative method for finding the approximate element of the common set of solutions of (1), (12), and (15) for a strictly pseudocontraction mapping in real Hilbert space, which can be viewed as a refinement and improvement of some existing methods for solving a system of variational inequality problem, a mixed equilibrium problem, and a hierarchical fixed point problem. It is easy to verify that Algorithm 11 includes some existing methods (e.g., $[6,11,17,31])$ as special cases. Therefore, the new algorithm is expected to be widely applicable.

\section{References}

[1] R. U. Verma, "Projection methods, algorithms, and a new system of nonlinear variational inequalities," Computers \& Mathematics with Applications, vol. 41, no. 7-8, pp. 1025-1031, 2001.

[2] R. U. Verma, "General convergence analysis for two-step projection methods and applications to variational problems," Applied Mathematics Letters, vol. 18, no. 11, pp. 1286-1292, 2005.

[3] G. Stampacchia, "Formes bilinéaires coercitives sur les ensembles convexes," Comptes Rendus de l'Acadmie des Sciences, vol. 258, pp. 4413-4416, 1964.

[4] G. L. Acedo and H.-K. Xu, "Iterative methods for strict pseudocontractions in Hilbert spaces," Nonlinear Analysis: Theory, Methods \& Applications, vol. 67, no. 7, pp. 2258-2271, 2007.

[5] E. Blum and W. Oettli, "From optimization and variational inequalities to equilibrium problems," The Mathematics Student, vol. 63, no. 1-4, pp. 123-145, 1994.

[6] F. Cianciaruso, G. Marino, L. Muglia, and Y. Yao, "On a two-step algorithm for hierarchical fixed point problems and variational inequalities," Journal of Inequalities and Applications, vol. 2009, Article ID 208692, 13 pages, 2009.
[7] L.-C. Ceng, C. Wang, and J.-C. Yao, "Strong convergence theorems by a relaxed extragradient method for a general system of variational inequalities," Mathematical Methods of Operations Research, vol. 67, no. 3, pp. 375-390, 2008.

[8] S. S. Chang, H. W. Joseph Lee, and C. K. Chan, "Generalized system for relaxed cocoercive variational inequalities in Hilbert spaces," Applied Mathematics Letters, vol. 20, no. 3, pp. 329-334, 2007.

[9] S. Chang, H. W. Joseph Lee, and C. K. Chan, "A new method for solving equilibrium problem fixed point problem and variational inequality problem with application to optimization," Nonlinear Analysis: Theory, Methods \& Applications, vol. 70, no. 9, pp. 3307-3319, 2009.

[10] S. D. Flåm and A. S. Antipin, "Equilibrium programming using proximal-like algorithms," Mathematical Programming, vol. 78, no. 1, pp. 29-41, 1997.

[11] G. Gu, S. Wang, and Y. J. Cho, "Strong convergence algorithms for hierarchical fixed points problems and variational inequalities," Journal of Applied Mathematics, vol. 2011, Article ID 164978, 17 pages, 2011.

[12] Z. Huang and M. A. Noor, "An explicit projection method for a system of nonlinear variational inequalities with different $(\gamma, r)$ cocoercive mappings," Applied Mathematics and Computation, vol. 190, no. 1, pp. 356-361, 2007.

[13] P. Katchang and P. Kumam, "A new iterative algorithm of solution for equilibrium problems, variational inequalities and fixed point problems in a Hilbert space," Journal of Applied Mathematics and Computing, vol. 32, no. 1, pp. 19-38, 2010.

[14] K. R. Kazmi and S. H. Rizvi, "A hybrid extragradient method for approximating the common solutions of a variational inequality, a system of variational inequalities, a mixed equilibrium problem and a fixed point problem," Applied Mathematics and Computation, vol. 218, no. 9, pp. 5439-5452, 2012.

[15] W. Kumam and P. Kumam, "Hybrid iterative scheme by a relaxed extragradient method for solutions of equilibrium problems and a general system of variational inequalities with application to optimization," Nonlinear Analysis: Hybrid Systems, vol. 3, no. 4, pp. 640-656, 2009.

[16] J.-L. Lions and G. Stampacchia, "Variational inequalities," Communications on Pure and Applied Mathematics, vol. 20, pp. 493519, 1967.

[17] P.-E. Maingé and A. Moudafi, "Strong convergence of an iterative method for hierarchical fixed-point problems," Pacific Journal of Optimization, vol. 3, no. 3, pp. 529-538, 2007.

[18] G. Marino and H.-K. Xu, "Convergence of generalized proximal point algorithms," Communications on Pure and Applied Analysis, vol. 3, no. 4, pp. 791-808, 2004.

[19] G. Marino and H.-K. Xu, "A general iterative method for nonexpansive mappings in Hilbert spaces," Journal of Mathematical Analysis and Applications, vol. 318, no. 1, pp. 43-52, 2006.

[20] G. Marino and H.-K. Xu, "Explicit hierarchical fixed point approach to variational inequalities," Journal of Optimization Theory and Applications, vol. 149, no. 1, pp. 61-78, 2011.

[21] A. Moudafi and M. Théra, "Proximal and dynamical approaches to equilibrium problems," in Ill-Posed Variational Problems and Regularization Techniques (Trier, 1998), vol. 477 of Lecture Notes in Economics and Mathematical Systems, pp. 187-201, Springer, Berlin, Germany, 1999.

[22] A. Moudafi, "Mixed equilibrium problems: sensitivity analysis and algorithmic aspect," Computers \& Mathematics with Applications, vol. 44, no. 8-9, pp. 1099-1108, 2002. 
[23] A. Moudafi, "Krasnoselski-Mann iteration for hierarchical fixed-point problems," Inverse Problems, vol. 23, no. 4, pp. 1635$1640,2007$.

[24] M. A. Noor and K. I. Noor, "Projection algorithms for solving a system of general variational inequalities," Nonlinear Analysis: Theory, Methods \& Applications, vol. 70, no. 7, pp. 2700-2706, 2009.

[25] S. Plubtieng and R. Punpaeng, "A general iterative method for equilibrium problems and fixed point problems in Hilbert spaces," Journal of Mathematical Analysis and Applications, vol. 336, no. 1, pp. 455-469, 2007.

[26] X. Qin, M. Shang, and Y. Su, "A general iterative method for equilibrium problems and fixed point problems in Hilbert spaces," Nonlinear Analysis: Theory, Methods \& Applications, vol. 69, no. 11, pp. 3897-3909, 2008.

[27] R. T. Rockafellar, "On the maximality of sums of nonlinear monotone operators," Transactions of the American Mathematical Society, vol. 149, pp. 75-88, 1970.

[28] R. U. Verma, "Generalized system for relaxed cocoercive variational inequalities and projection methods," Journal of Optimization Theory and Applications, vol. 121, no. 1, pp. 203210, 2004

[29] H.-K. Xu, "Iterative algorithms for nonlinear operators," Journal of the London Mathematical Society, vol. 66, no. 1, pp. 240-256, 2002.

[30] H. Yang, L. Zhou, and Q. Li, "A parallel projection method for a system of nonlinear variational inequalities," Applied Mathematics and Computation, vol. 217, no. 5, pp. 1971-1975, 2010.

[31] Y. Yao, Y. J. Cho, and Y.-C. Liou, "Iterative algorithms for hierarchical fixed points problems and variational inequalities," Mathematical and Computer Modelling, vol. 52, no. 9-10, pp. 1697-1705, 2010.

[32] Y. Yao, Y.-C. Liou, and S. M. Kang, "Approach to common elements of variational inequality problems and fixed point problems via a relaxed extragradient method," Computers \& Mathematics with Applications, vol. 59, no. 11, pp. 3472-3480, 2010.

[33] H. Zhou, "Convergence theorems of fixed points for $\kappa$-strict pseudo-contractions in Hilbert spaces," Nonlinear Analysis: Theory, Methods \& Applications, vol. 69, no. 2, pp. 456-462, 2008. 


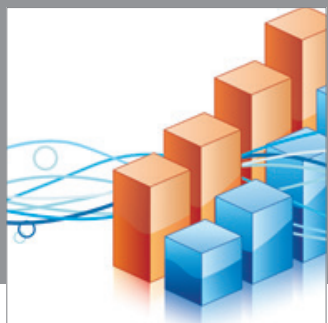

Advances in

Operations Research

mansans

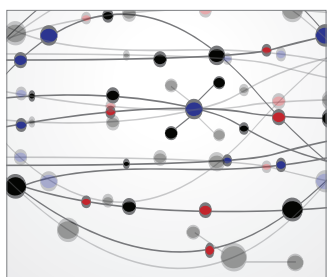

The Scientific World Journal
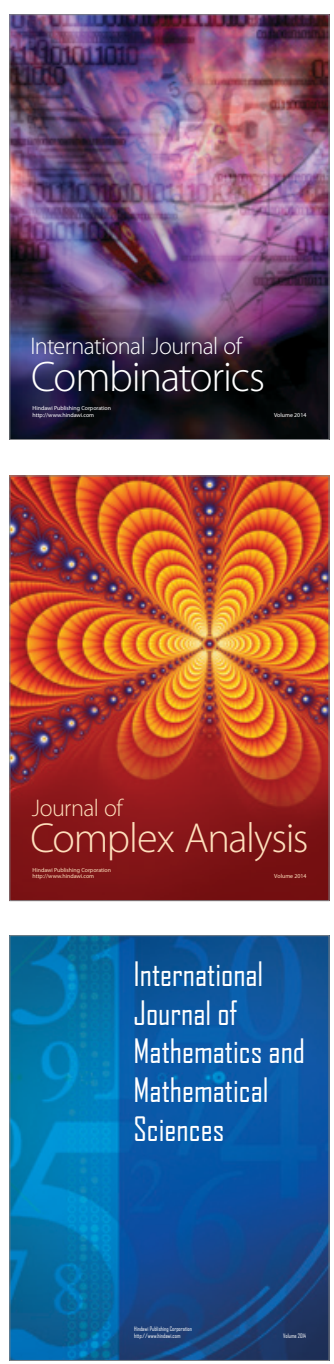
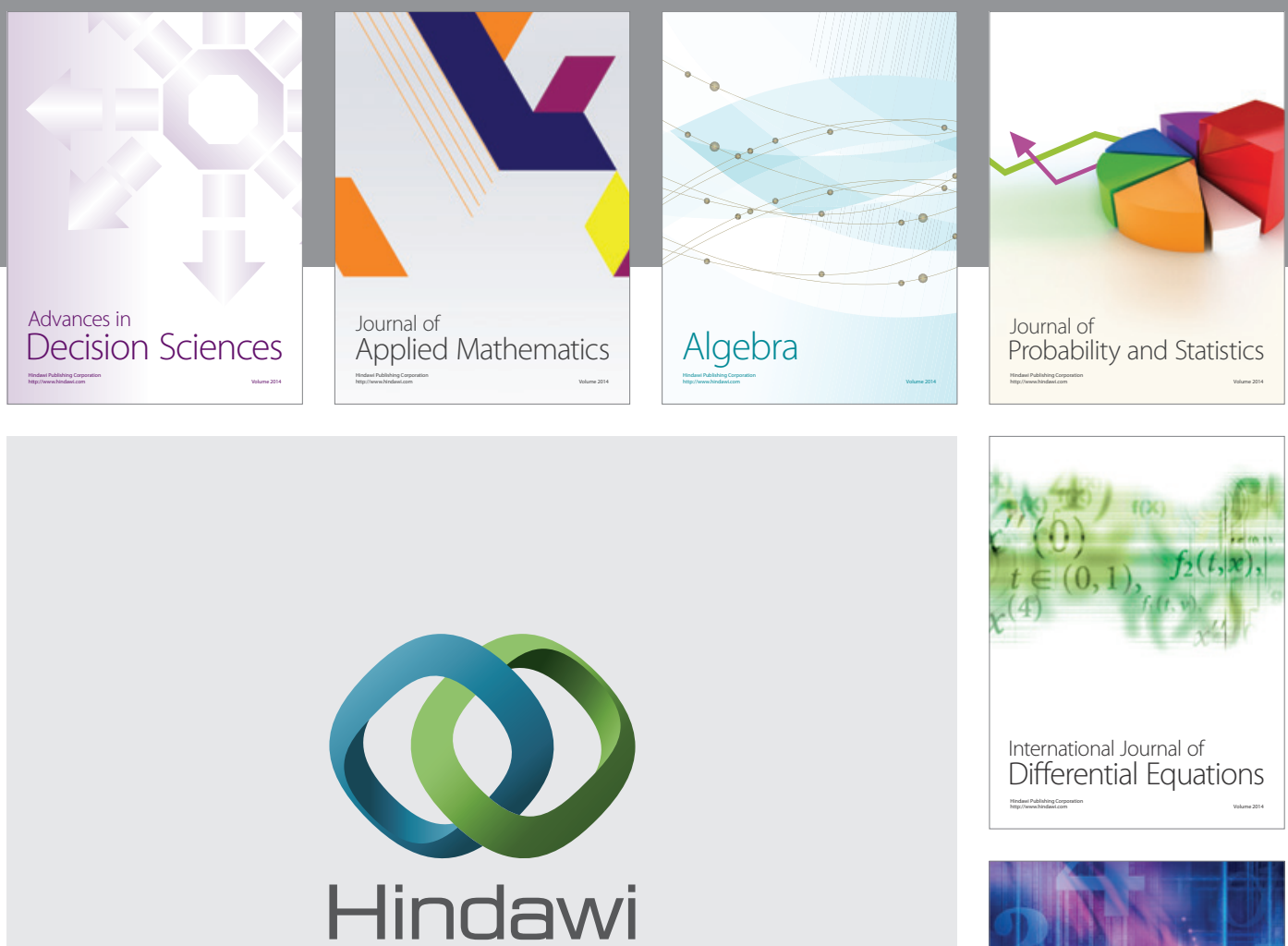

Submit your manuscripts at http://www.hindawi.com
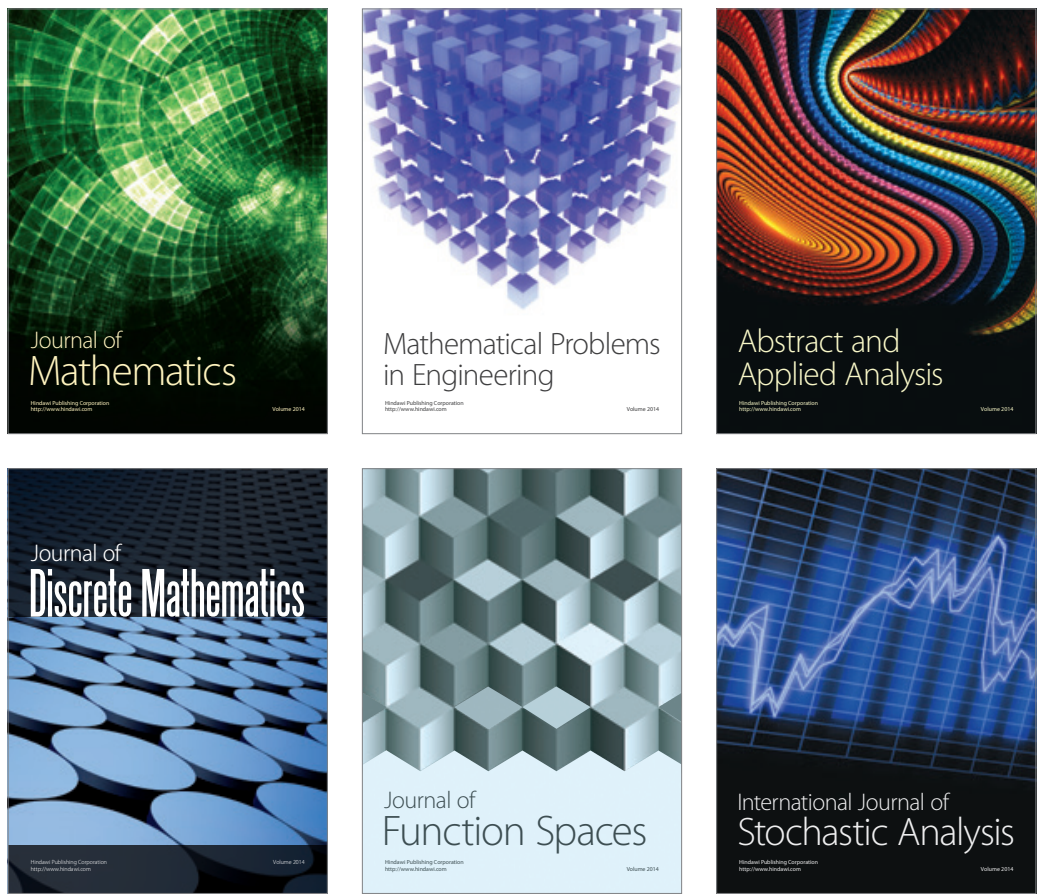

Journal of

Function Spaces

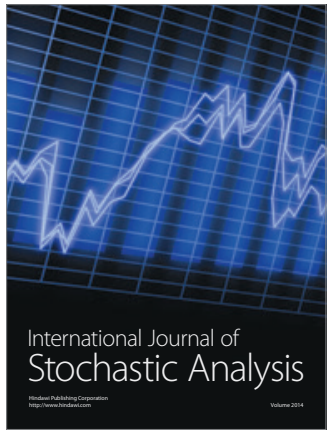

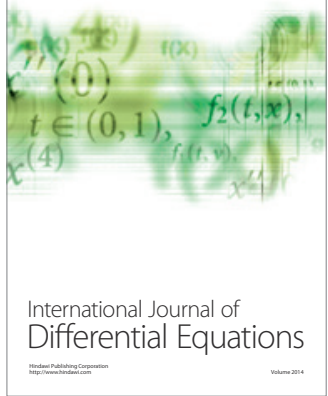
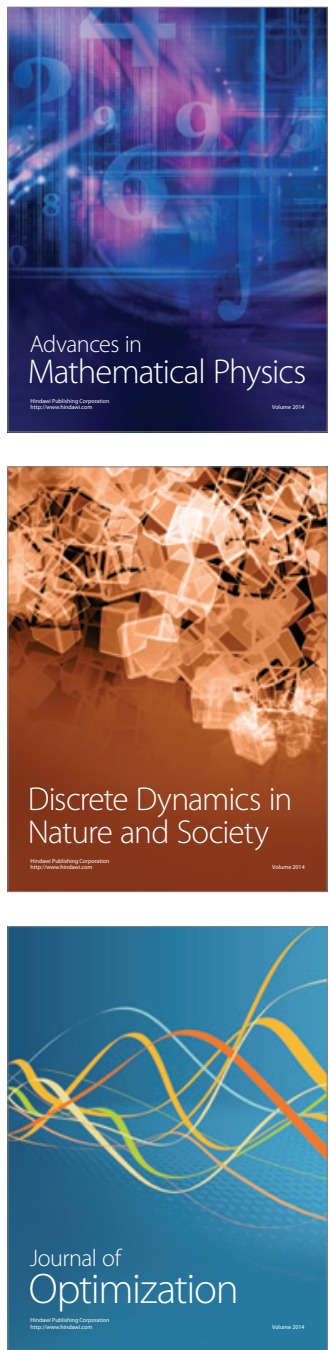